\title{
A new X-ray-selected sample of very extended galaxy groups from the ROSAT All-Sky Survey
}

\author{
Weiwei $\mathrm{Xu}^{1,2,3}$, Miriam E. Ramos-Ceja ${ }^{2}$, Florian Pacaud ${ }^{2}$, Thomas H. Reiprich ${ }^{2}$, and Thomas Erben ${ }^{2}$ \\ 1 National Astronomical Observatories, Chinese Academy of Sciences, Beijing, PR China \\ e-mail: weiweixu@bao.ac.cn \\ 2 Argelander-Insitut für Astronomie, Universität Bonn, Bonn, Germany \\ ${ }^{3}$ University of Chinese Academy of Sciences, Beijing, PR China
}

Received 20 March 2018 / Accepted 3 September 2018

\begin{abstract}
Context. Some indications for tension have long been identified between cosmological constraints obtained from galaxy clusters and primary cosmic microwave background (CMB) measurements. Typically, assuming the matter density and fluctuations, as parameterized with $\Omega_{\mathrm{m}}$ and $\sigma_{8}$, estimated from CMB measurements, many more clusters are expected than those actually observed. This has been reinforced recently by the Planck collaboration. One possible explanation could be that certain types of galaxy groups or clusters were missed in samples constructed in previous surveys, resulting in a higher incompleteness than estimated.

Aims. In this work, we aim to determine if a hypothetical class of very extended, low-surface-brightness galaxy groups or clusters have been missed in previous X-ray cluster surveys based on the ROSAT All-Sky Survey (RASS).

Methods. We applied a dedicated source-detection algorithm sensitive also to more unusual group or cluster surface-brightness distributions. It includes a multiresolution filtering, a source-detection algorithm, and a maximum-likelihood fitting procedure. To optimize parameters, this algorithm is calibrated using extensive simulations before it is used to reanalyze the RASS data. In addition, the cross-correlation of the candidates with optical/infrared surveys is used for cluster identification and redshift estimation.

Results. We found many known groups but also a number of new group candidates, which are not included in any previous X-ray or SZ cluster catalogs. In this paper, we present a pilot sample of 13 very extended groups discovered in the RASS at positions where no X-ray source has been detected previously and with clear optical counterparts. The X-ray fluxes of at least 5 of these are above the nominal flux-limits of previous RASS cluster catalogs $\left(~ \gtrsim 3 \times 10^{-12} \mathrm{erg} \mathrm{s}^{-1} \mathrm{~cm}^{-2}\right.$ in the $0.1-2.4 \mathrm{keV}$ energy band). They have low mass $\left(10^{13} M_{\odot} \lesssim M_{500} \lesssim 10^{14} M_{\odot}\right.$; i.e., they are galaxy groups), are at low redshift $(z<0.08)$, and exhibit flatter surface-brightness distributions than usual.

Conclusions. We demonstrate that galaxy groups were missed in previous RASS surveys, possibly due to the flat surface-brightness distributions of this potential new population. Analysis of the full sample will show if this might have a significant effect on previous cosmological parameter constraints based on RASS cluster surveys.
\end{abstract}

Key words. galaxies: clusters: general - galaxies: groups: general - galaxies: clusters: intracluster medium large-scale structure of Universe - X-rays: galaxies: clusters

\section{Introduction}

Groups and clusters of galaxies are powerful probes to test and constrain cosmological models, independent of and complementary to other methods (e.g., Allen et al. 2011), such as Type Ia supernovae (Kowalski et al. 2008) and the cosmic microwave background (CMB; Dunkley et al. 2009). These systems have undergone gravitational relaxation and therefore are the largest objects whose masses can be measured with several independent methods. These measurements allow us to use the total matter content of clusters, and cluster counts at different epochs, to constrain the amount of dark matter in the universe. Moreover, the rate of gravitationally driven structure formation, as traced by the evolution of the cluster population, provides independent constraints on the amount and properties of dark energy in the universe.

Most cosmological studies that use galaxy groups and clusters require well-defined, large cluster samples. To this purpose, cluster samples have to be carefully treated by understanding their underlying cluster populations as well as their cluster mass determination and calibration. This has become clear again in the recently identified tension between primary CMB and cluster count constraints on cosmological parameters encountered by the Planck collaboration (Planck Collaboration XX 2014; Planck Collaboration XXIV 2016b). Observational bias and the survey strategy must be carefully accounted for in order to provide a sufficiently accurate description of the observed cluster sample (e.g., Mantz et al. 2010; Pacaud et al. 2016; Schellenberger \& Reiprich 2017).

Early on, some tension was found between the values for $\sigma_{8}$, parameterizing the matter fluctuation amplitude, and $\Omega_{\mathrm{m}}$, the mean normalized matter density. Cluster studies preferred $\sigma_{8} \sim 0.7$ (e.g., Borgani et al. 2001; Reiprich \& Böhringer 2002; Seljak 2002; Viana et al. 2002) while CMB studies showed $\sigma_{8} \sim 0.9$ (e.g., Spergel et al. 2003) for a given $\Omega_{\mathrm{m}} \sim 0.3$. After some oscillations (e.g., Spergel et al. 2007; Henry et al. 2009), now both probes seem to have settled around $\sigma_{8} \sim 0.8$ (e.g., Mantz et al. 2010; Hinshaw et al. 2013; de Haan et al. 2016; Planck Collaboration XXIV 2016b; Schellenberger \& Reiprich 2017). Still, cluster constraints tend to be on the lower $\sigma_{8}$ side compared to primary CMB constraints while the statistical uncertainties shrink. This slight 
offset may simply reflect statistical uncertainty but could also be due to interesting systematic, physical, or cosmological effects (e.g., Schellenberger \& Reiprich 2017; McCarthy et al. 2018). Another probe at low redshift, that is, cosmic shear, also appears to result in lower $\sigma_{8}$ estimations (e.g., Abbott et al. 2016; Hildebrandt et al. 2017).

Among the observational techniques for detection and analysis of galaxy groups and clusters, X-ray imaging is one of the most sensitive and reliable. The presence of the hot intra-cluster gas ensures that only genuine gravitationally bound structures are included, leading to highly pure and well-understood cluster samples in X-ray surveys.

The ROentgen SATellit (ROSAT) observatory has been the first and the only imaging telescope to perform an X-ray All-Sky Survey (RASS, Truemper 1992, 1993). The ROSAT mission was able to detect $\sim 125000$ X-ray sources (Voges et al. 1999, 2000; Boller et al. 2016), providing an ideal basis for the construction of a large X-ray cluster sample for cosmological studies. In the northern hemisphere the largest and most representative galaxy cluster compilations from contiguous areas of RASS include the Bright Cluster Sample (BCS; Ebeling et al. 1998), which is a flux-limited sample (flux $\geq 4.4 \times 10^{-12} \mathrm{erg} \mathrm{s}^{-1} \mathrm{~cm}^{-2}$ in the $0.1-2.4 \mathrm{keV}$ energy band), the extended BCS, which is a lowflux extension of BCS (eBCS, flux $\geq 2.8 \times 10^{-12} \mathrm{erg} \mathrm{s}^{-1} \mathrm{~cm}^{-2}$ in the 0.1-2.4 keV energy band, Ebeling et al. 2000), the Northern ROSAT All-Sky Survey (NORAS; Böhringer et al. 2000), which is not flux-limited and its selection is based on a minimum count rates $\left(0.06 \mathrm{cnts} \mathrm{s}^{-1}\right.$ in the $0.1-2.4 \mathrm{keV}$ energy band, roughly corresponding to a flux $1.2 \times 10^{-12} \mathrm{erg} \mathrm{s}^{-1} \mathrm{~cm}^{-2}$ in the same band, see their Fig. 8a), and a source extent likelihood. The southern hemisphere has the ROSAT-ESO Flux Limited X-ray Galaxy Cluster Survey (REFLEX I - Böhringer et al. 2001), which is a flux-limited sample (flux $\geq 3 \times$ $10^{-12} \mathrm{erg} \mathrm{s}^{-1} \mathrm{~cm}^{-2}$ in the $0.1-2.4 \mathrm{keV}$ energy band).

However, these galaxy cluster samples obtained from the RASS data could be incomplete, because the sliding cell algorithm (Harnden et al. 1984), the detection method for most of such cluster samples, works most efficiently at finding pointlike sources, instead of very extended sources with low-surfacebrightness, such as galaxy groups and clusters at low redshift (e.g., Valtchanov et al. 2001). In addition, the inhomogeneous sky coverage of RASS might make the detection more difficult in regions with low or heavily varied exposure time. If the true incompleteness is higher than the estimated one, biased constraints on cosmological parameters may result. For studies of cluster mass function, this would tend to lower the inferred values of $\Omega_{\mathrm{m}}$ and/or $\sigma_{8}$; see, for example, the tests carried out in Schellenberger \& Reiprich (2017), shown in Fig. 10 therein.

The main goal of this work is to look for possible missing extended galaxy groups or clusters and assess the completeness of the RASS-based cluster catalogs. For this purpose we reanalyze the RASS images ${ }^{1}$ in the $0.5-2.0 \mathrm{keV}$ energy band, with a wavelet-based source-detection algorithm combined with a maximum-likelihood fitting method to detect and characterize $\mathrm{X}$-ray sources. This procedure is chosen based on its successful performance in finding extended X-ray sources with low surfacebrightness, that is, galaxy groups and clusters at low redshift, in various X-ray surveys (e.g., Rosati et al. 1995; Vikhlinin et al. 1998; Pacaud et al. 2006, Lloyd-Davies et al. 2011). In this initial paper, we discuss the general X-ray characteristics of the possible missing groups and clusters in ROSAT catalogs, and

\footnotetext{
1 http://www . xray.mpe.mpg.de/cgi-bin/rosat/ rosat-survey
}

show the properties of a pilot sample of confirmed detections. We refer to our extended detections as cluster candidates until we present the measured masses and can determine if they are galaxy groups or clusters. Subsequently, we refer to groups with $M_{500}<10^{14} M_{\odot}$ as galaxy groups and those with larger masses as galaxy clusters.

Throughout this paper, a Hubble constant $H_{0}=$ $70 \mathrm{~km} \mathrm{~s}^{-1} \mathrm{Mpc}^{-1}$, matter density parameter $\Omega_{\mathrm{m}}=0.3$, and dark energy density parameter $\Omega_{\Lambda}=0.7$ are assumed unless stated otherwise. Moreover, the images used in this paper as well as the count rates are in the $0.5-2.0 \mathrm{keV}$ energy band, while the fluxes are expressed in the $0.1-2.4 \mathrm{keV}$ energy band unless stated otherwise.

\section{Cluster-detection method}

As stated in Rosati et al. (1995), wavelet transformation is capable of detecting faint X-ray sources with a variety of sizes and surface-brightnesses. Vikhlinin et al. (1998) showed that a further implementation of a maximum-likelihood method enhances the characterization of extended sources from the waveletdetected sources. The combination of these methods has resulted in successful galaxy cluster catalogs using XMM-Newton data in recent years (e.g., Pacaud et al. 2006; Lloyd-Davies et al. 2011). Given that our main goal is to find possible missing extended groups and clusters with low surface-brightness in the RASS data, we follow the general scheme and implement a detection pipeline similar to the one used in Pacaud et al. (2006; hereafter P06), proceeding in three main steps:

1. The Poisson noise is first removed from the images with a multiresolution filtering procedure.

2. The filtered images are scanned by a source-detection algorithm, resulting in a preliminary source list.

3. Each detected source is fitted and characterized by a maximum-likelihood procedure.

These steps are first applied to the simulations described in Sect. 3, with the aim to assess the reliability of this method in finding extended clusters with low surface-brightness; this approach is then applied to the RASS observations. In the following, a brief description of each step is presented.

The simulated images are first filtered using a wavelet algorithm designed to handle Poisson data. It is a completely new implementation of the wavelet task used in P06, MR_FILTER ${ }^{2}$. Like its predecessor, it relies on a "à trous" ("with holes") transform with a cubic B-spline wavelet (also known as starlet transform) and filters the noise by imposing hard thresholds on the wavelet coefficients, estimated from autoconvolution histograms (see Starck \& Pierre 1998, for a detailed description). A smoothed and denoised image is then reconstructed iteratively from the significant coefficients. A key feature of the new implementation is the ability to account for varying exposure time not only in the reconstruction, but also for the determination of the significance thresholds. More details on the software are provided in Faccioli et al. (2018).

The source detection on the filtered images is performed by the SExtractor ${ }^{3}$ software (Bertin \& Arnouts 1996). Originally, SExtractor was developed to detect objects from optical data, but it can also be applied to the multiresolution filtered X-ray images, since the filtering removes most of the noise from it to produce a smooth background. This step proceeds exactly as in

\footnotetext{
2 From the MR/1 package: http://www.multiresolutions.com/ $\mathrm{mr} /$

3 http://WwW.astromatic.net/software/sextractor
} 
Table 1. Summary of the extended source simulations in the ROSAT-like images.

\begin{tabular}{|c|c|c|c|c|c|c|c|c|c|c|}
\hline \multirow{2}{*}{$\begin{array}{l}\text { Input flux }(0.5-2.0 \mathrm{keV}) \\
\left(\mathrm{erg} \mathrm{s}^{-1} \mathrm{~cm}^{-2}\right)\end{array}$} & \multicolumn{10}{|c|}{ Input core radius } \\
\hline & $0.75^{\prime}$ & $1.50^{\prime}$ & $2.25^{\prime}$ & $3.00^{\prime}$ & $3.75^{\prime}$ & $4.50^{\prime}$ & $5.25^{\prime}$ & $6.00^{\prime}$ & $12.00^{\prime}$ & $24.00^{\prime}$ \\
\hline $1 \times 10^{-12}$ & 20 & 20 & 20 & 20 & 20 & 20 & 20 & 20 & 25 & 100 \\
\hline $3 \times 10^{-12}$ & 20 & 20 & 20 & 20 & 20 & 20 & 20 & 20 & 25 & 100 \\
\hline $5 \times 10^{-12}$ & 20 & 20 & 20 & 20 & 20 & 20 & 20 & 20 & 25 & 100 \\
\hline $1 \times 10^{-11}$ & 20 & 20 & 20 & 20 & 20 & 20 & 20 & 20 & 25 & 100 \\
\hline $5 \times 10^{-11}$ & 20 & 20 & 20 & 20 & 20 & 20 & 20 & 20 & 25 & 100 \\
\hline
\end{tabular}

Notes. The column values indicate the number of simulated images for a given $\beta$ value. For each set of parameters $\left(\beta, r_{\mathrm{c}}\right.$, and flux within $\left.r_{\mathrm{c}}\right), 100$ clusters are simulated. For example, a value of 20 in the table means that there are 5 clusters in one simulated image for a given set of parameters. Simulations were performed for an exposure time of $450 \mathrm{~s}$ with a background level of $0.08 \mathrm{cnts}_{\text {pixel }}{ }^{-1}$, and four $\beta$ values $(0.40,0.55,0.66$, and $0.70)$.

P06 and the most relevant SExtractor parameters are given in their Table 1 . The only difference is the pixel size, which we set to 45 arcsec.

In the final step of the detection procedure, the sources identified by SExTRACTOR are characterized by a maximumlikelihood profile-fitting algorithm (also a new implementation compared to P06). Starting from the SExTRactor measurements as a guess for the source properties, it maximizes the Poisson likelihood (C-statistic, Cash 1979) for each source individually, masking surrounding detections based on the SExTRACTOR segmentation map. The best fit model parameters are derived using the simplex minimization method AMOEBA (Press et al. 1992). The significance of each detection (hereafter detection likelihood) is then quantified using a relation based on the best-fit likelihood as:

DET_LIKE $=2\left[\log \left(\mathcal{L}_{\text {bgd }}\right)-\log \left(\mathcal{L}_{\text {ext }}\right)\right]$,

where $\mathcal{L}_{\text {bgd }}$ and $\mathcal{L}_{\text {ext }}$ are respectively the Poisson likelihoods of the best background (flat distribution) and extended source model. The extended source model is given by a $\beta$ profile, with a $\beta$ value of two thirds (see Eq. (4)). A significance of the source extension (the extension likelihood) is also provided as:

EXT_LIKE $=2\left[\log \left(\mathcal{L}_{\text {pnt }}\right)-\log \left(\mathcal{L}_{\text {ext }}\right)\right]$,

where $\mathcal{L}_{\text {pnt }}$ is the likelihood of the point-source model. It is worth mentioning that the point-like and the extended source models are convolved with the averaged ROSAT PSF. Aside from the instrument specifics, the two main differences compared to P06 lie in the definition of the extension / detection likelihoods (which included an analytical correction for the number of fitted parameters in P06) and the background treatment. Indeed, our model includes a constant sky background to be fitted, whereas it was set in P06 through an analytical maximization of the likelihood with respect to the model normalization.

\section{Image simulations}

We first assess our cluster-detection analysis on image simulations. For this, we perform extensive and dedicated Monte Carlo simulations of ROSAT images. Our procedure creates images with point-like and extended sources, which represent active galactic nuclei (AGNs) and galaxy clusters, respectively. It also takes into account the main ROSAT instrumental characteristics (such as point spread function (PSF), background, and Poisson noise). In this section, we describe the setup of our simulations.

\subsection{General parameters}

We simulate images with the same size as the publicly available RASS tiles, that is, $6.40 \times 6.40 \mathrm{deg}^{2}$, with a pixel size of 45 arcsec.

The input sources are convolved with the survey averaged PSF at $1.0 \mathrm{keV}$ (as appropriate for the $0.5-2.0 \mathrm{keV}$ energy band that we are simulating). The PSF itself is obtained from the Gaussian model of Boese (2000). This model describes the width of the PSF (in units of arcsec) as a function of energy and offaxis:

$\sigma(E, \epsilon)=\sqrt{108.7 E^{-0.888}+1.121 E^{6}+0.219 \epsilon^{2.848}}$,

where $E$ is the energy expressed in $\mathrm{keV}$ and $\epsilon$ is the off-axis angle in arcmin. For the field averaged PSF, applicable to survey observation, we generate Gaussian PSF at different off-axis angles and weight them according to the telescope vignetting and detector area. The resulting PSF has a full width at half maximum (FWHM) of $\sim 45$ arcsec and, despite originating from a sum of Gaussian functions, has much broader wings than a single Gaussian.

The ROSAT survey exposure distribution is not uniform. It varies from 100 to $40000 \mathrm{~s}$ at the ecliptic equator and poles, respectively (Voges et al. 1999). Given that the purpose of this work is to characterize the average potential of our extended source-detection algorithm and to provide insights into the properties of new clusters, we adopt a flat exposure time of $450 \mathrm{~s}$ for the simulations. This value is the average obtained over all exposure maps of the ROSAT all-sky survey. In a following paper, the limitations of this approach will be fully discussed.

We also include a flat X-ray background with a value of $0.08 \mathrm{cnts} \mathrm{pixel}^{-1}$. This value is calculated by averaging the background maps of the ROSAT all-sky survey. These maps are created by the SExTRACTOR software (see Sect. 2 for further details).

\subsection{Galaxy clusters and AGNs}

The surface-brightness, $S_{\mathrm{X}}$, of galaxy clusters is described by the spherically symmetric $\beta$ model (Cavaliere \& Fusco-Femiano 1976); it is given by

$S_{\mathrm{X}}(r) \propto\left[1+\left(\frac{r}{r_{\mathrm{c}}}\right)^{2}\right]^{-3 \beta+1 / 2}$,

where $r_{\mathrm{c}}$ is the core radius of the cluster, and $\beta$ determines the slope of the brightness profile. Different values for $\beta, r_{\mathrm{c}}$, and flux 


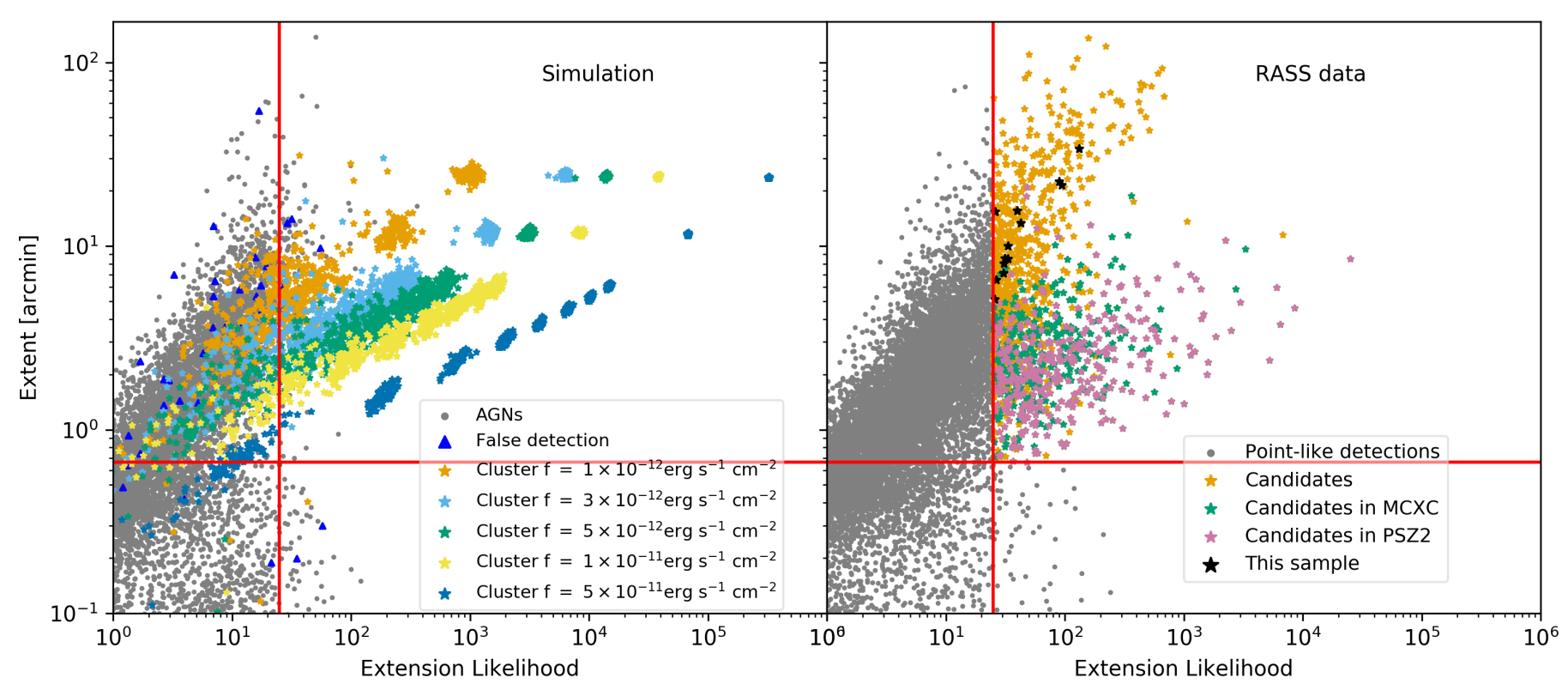

Fig. 1. Selection criteria for extended sources. The selection is performed in the extension likelihood - extent plane. The red-solid lines define the optimal parameters obtained from simulations to characterize extended sources. Left: simulation results. Gray dots represent simulated AGNs, and blue triangles, false detections. Colored stars represent clusters with different input fluxes. Right: results from reprocessing RASS data. Gray dots stand for point-like detections and the star symbols for the cluster candidates. Green and pink stars show the candidates with identified counterparts in the MCXC and PSZ2 catalogs, respectively. The black stars represent the 13 groups of our pilot sample described in Table 3.

(see Table 1) are used in order to cover a wide range of possible cluster morphologies. For each set of parameters, 100 clusters are simulated with controlled positions. In order to reduce the overlap of X-ray emission from the simulated clusters, the distances between them are larger than $2 \times r_{500}$. Moreover, the cluster distances to the nearest image edge are always larger than $r_{500}$. The value of $r_{500}$ is taken as $7 \times r_{\mathrm{c}}$.

Since AGNs represent the majority of extragalactic X-ray sources, a population of them are included in the image simulations. The surface-brightness of AGNs is described by the ROSAT PSF. The AGN flux distribution and source density are obtained using the $\log N-\log S$ from Moretti et al. (2003) down to a flux of $2.29 \times 10^{-14} \mathrm{erg} \mathrm{s}^{-1} \mathrm{~cm}^{-2}$. The spatial distribution of AGNs is assumed to be random.

To convert the flux of clusters and AGNs into photon count, PIMMS $^{4}$ is used. The energy conversion factor used for clusters is $1.12 \times 10^{-11}\left(\mathrm{erg} \mathrm{s}^{-1} \mathrm{~cm}^{-2}\right) /\left(\mathrm{cnts} \mathrm{s}^{-1}\right)$, which is obtained by assuming an absorbed APEC model with $n_{\mathrm{H}}=5.95 \times 10^{20} \mathrm{~cm}^{-2}$, an abundance of 0.4 times solar abundance, a temperature of $2.73 \mathrm{keV}\left(10^{7.5} \mathrm{~K}\right)$, and $z=0.10$. The galactic neutral hydrogen column density value, $n_{\mathrm{H}}$, is taken from the average of all observations, excluding only a $\pm 20 \mathrm{deg}$ area around the Galactic plane. The values of the temperature and redshift are chosen based on our objective of detecting relatively low-temperature and nearby clusters that were previously missed. We note that the energy conversion factor changes by less than $3 \%$ for $0.02<z<$ 0.18 and $T=2.73 \mathrm{keV}$. The energy conversion factor for AGNs is $1.15 \times 10^{-11}\left(\mathrm{erg} \mathrm{s}^{-1} \mathrm{~cm}^{-2}\right) /\left(\mathrm{cnts} \mathrm{s}^{-1}\right)$. We obtain this factor by assuming an absorbed power-law model with photon index equal to 1.85 , which is roughly the peak of the observed distribution for X-ray AGNs (Ueda et al. 2014), and with the same $n_{\mathrm{H}}$ value as before.

\footnotetext{
4 https://heasarc.gsfc.nasa.gov/cgi-bin/Tools/ w3pimms/w3pimms.pl
}

\subsection{Source classification using simulations}

All simulated images were processed with the pipeline described in Sect. 2. The positions of the detected sources were crossidentified with the simulation inputs using a correlation radius of 6 arcmin for point-like sources and $3.5 \times r_{\mathrm{c}}$ for extended sources. We use a variable matching radius for extended sources because their positional accuracy is size dependent.

Following P06, we explore the parameter space of the maximum-likelihood fitting method to place source classification criteria. The most relevant parameters are: detection likelihood, extension likelihood, source extent (i.e., core radius), and source position.

The wavelet image reconstruction creates artifacts near the image edges, which result in false detections. Therefore, we exclude all detected sources located close to the image edges ( $<15$ pixels to image sides). Moreover, our analysis shows that a threshold value of 20 in the detection likelihood removes $95.4 \%$ of the false detections, that is, sources that are not simulated but are found by the detection algorithm. Although this threshold removes $50.8 \%$ of the detected AGNs, it preserves $96.7 \%$ of the detected clusters. We obtain an average of $\sim 1.5$ false detections per simulated image. The number of 1.5 false detections is a global value of the number of false detections regardless of the origin of the simulated source (point-like or extended). However, as discussed in the following, this number is mainly generated by false point-like detections.

By scanning the extension likelihood - extent parameter space, we look for a location where the majority of our simulated extended sources are recovered while maintaining the contamination from point-like sources at a low level. The lefthand panel of Fig. 1 shows the main selection criteria for extended sources with $\beta=2 / 3$ in this plane. This is given by values of extension likelihood $>25$ and extent $>0.67$ arcmin. The discrete distribution in the extent values reflects the different input core radius values used in the simulations (see Table. 1). Using these extended selection criteria we obtain 


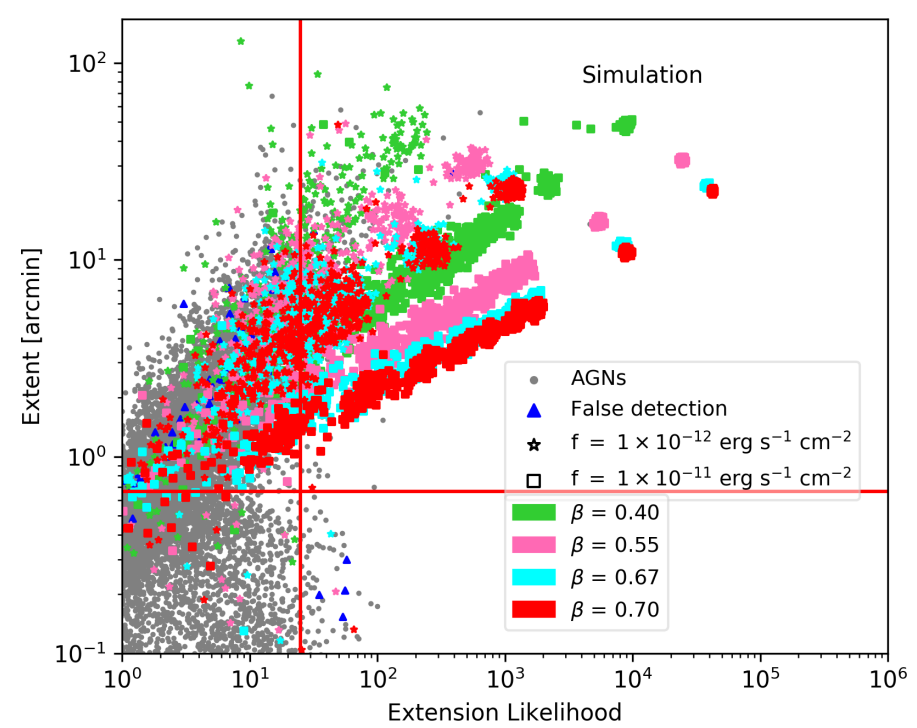

Fig. 2. Similar plot as the left-hand panel of Fig. 1 showing the selection criteria for extended sources in the extension likelihood - extent plane. Gray dots represent simulated AGNs, and blue triangles, false detections. Colored symbols represent clusters with different input $\beta$ values. Filled stars show clusters with input flux $1 \times 10^{-12} \mathrm{erg} \mathrm{s}^{-1} \mathrm{~cm}^{-2}$, while filled squares are clusters with input flux $1 \times 10^{-11} \mathrm{erg} \mathrm{s}^{-1} \mathrm{~cm}^{-2}$.

$\sim 4.2 \times 10^{-3}$ extended false detections per simulated image, and $\sim 0.1$ misclassified AGNs per simulated image, that is, simulated AGN with values of extension likelihood $>25$ and extent $>0.67$ arcmin.

The minimum extent value chosen for extended source characterization, 0.67 arcmin, is slightly lower than the minimum value of core radius used in the simulations $(0.75 \mathrm{arcmin})$. We have tested the impact of this minimum extent value, by simulating clusters with $r_{\mathrm{c}}=0.5 \mathrm{arcmin}$. We found that these clusters are characterized by our algorithm with low values of extension likelihood, $<25$. Therefore, they are not characterized as extended sources. This does not represent an issue in the present work since our goal is to look for very extended galaxy clusters that might have been missed in previous studies.

Fig. 2 shows that the selection criteria are very similar for extended sources simulated with $\beta$ values different from two thirds. In contrast with the left-hand panel of Fig. 1, this plot displays only two values of input cluster fluxes, $1 \times 10^{-12} \mathrm{erg} \mathrm{s}^{-1} \mathrm{~cm}^{-2}$ and $1 \times 10^{-11} \mathrm{erg} \mathrm{s}^{-1} \mathrm{~cm}^{-2}$. In general, clusters simulated with small $\beta$ values tend to have lower extension-likelihood values and larger extent values.

The detection and characterization efficiency of the total number of simulated clusters can be summarized as follows. Approximately $34 \%$ of simulated clusters with $\beta=0.40$, flux equal to $1 \times 10^{-12} \mathrm{erg} \mathrm{s}^{-1} \mathrm{~cm}^{-2}$, and $r_{\mathrm{c}}>5^{\prime}$ are detected and characterized as extended sources. This percentage increases to $\sim 97 \%$ for clusters with flux equal to $3 \times 10^{-12} \mathrm{erg} \mathrm{s}^{-1} \mathrm{~cm}^{-2}$. About $69 \%$ of simulated clusters with $\beta=0.55$, flux equal to $1 \times 10^{-12} \mathrm{erg} \mathrm{s}^{-1} \mathrm{~cm}^{-2}$, and $r_{\mathrm{c}}>5^{\prime}$ are detected and characterized as extended sources. Broadly speaking, simulated clusters with fluxes $\geq 5 \times 10^{-12} \mathrm{erg} \mathrm{s}^{-1} \mathrm{~cm}^{-2}, \beta \geq 0.55$, and $r_{\mathrm{c}} \geq 5^{\prime}$ have $100 \%$ probability of being detected and characterized as extended sources. The full analysis of the completeness of our sample will be presented in a subsequent publication.
Table 2. Overview of the publications used to cross-check if cluster candidates have previously been detected.

\begin{tabular}{ll}
\hline \hline X-ray catalogs & Ebeling et al. (1996) \\
& Mehrtens et al. (2012) \\
& Liu et al. (2015) \\
& Pacaud et al. (2016) \\
& Wen et al. (2018) \\
\hline Optical catalogs & Zwicky \& Kowal (1968) \\
& Abell et al. (1989) \\
& Wen et al. (2009) \\
& Wen et al. (2012) \\
& Rykoff et al. (2014) \\
& Rykoff et al. (2016) \\
& Oguri et al. (2018) \\
\hline Microwave catalogs & Marriage et al. (2011) \\
& Hasselfield et al. (2013) \\
& Hilton et al. (2018) \\
\hline Galaxy catalogs & Zwicky \& Kowal (1968) \\
& Sinnott (1988) \\
\hline
\end{tabular}

\section{Results}

\subsection{Performance on real data}

Having calibrated the cluster candidate selection criteria on simulations, we proceed to run the detection algorithm on the real RASS data. The RASS data consist of 1378 image tiles, with varying exposure time (see Sect. 3). We exclude the data close to the plane of the Milky Way, the Magellanic Clouds, and the Virgo cluster regions (see Table 2 of Reiprich \& Böhringer 2002).

The right-hand panel of Fig. 1 shows the results of processing the RASS data. Applying the same criteria for extended sources as in Sect. 3.3, we find 1308 cluster candidates. In order to validate the authenticity of these cluster candidates, we first cross-identify them with well-known cluster catalogs.

Using a correlation radius of $10 \mathrm{arcmin}$, we match our cluster candidates with the Meta-Catalog of X-Ray Detected Clusters of Galaxies (MCXC; Piffaretti et al. 2011) and the second Planck catalog of Sunyaev-Zeldovich sources (PSZ2; Planck Collaboration XXVII 2016a), excluding clusters in the regions where we do not process the RASS data. The size of the correlation radius is determined from the distribution of the positional difference between our cluster candidates and the MCXC and PSZ2 clusters. Within 10 arcmin, the differential number of matched clusters decreases rapidly. Beyond this value, the positional difference distribution starts to rise, meaning that random matches start to appear due to the increasing area.

First, we correlate all our detections (i.e., regardless of their extent, detection likelihood, and extension likelihood values) with clusters of the MCXC and PSZ2 catalogs. We are able to detect $86.5 \%$ of the MCXC clusters. This percentage increases to $99.7 \%$ if we only select RASS-based MCXC clusters. Fig. 3 shows the redshift and $r_{500}$ (taken from Piffaretti et al. 2011) distributions of the matched and unmatched RASS-based MCXC clusters with our detected sources. From Fig. 3 we can see that we do not detect three RASS-based MCXC clusters. Two of such clusters are located in regions with exposure time $<70 \mathrm{~s}$, while the third one is positioned close to the ROSAT North Ecliptic Pole where the large gradient of exposure time may affect the positional accuracy of the detected sources in the region (our nearest detection is 12 arcmin away). We also observe a high 

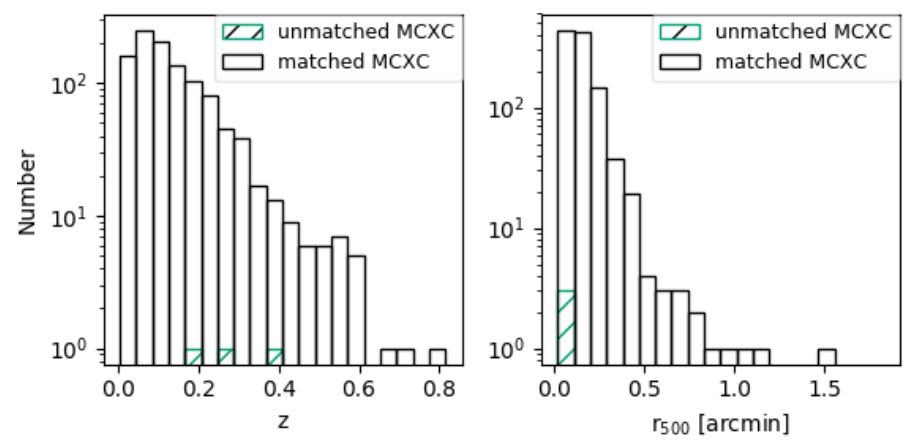

Fig. 3. Distribution of redshift and $r_{500}$ of matched and unmatched RASS-based MCXC clusters with our detected sources. The $y$-axis is a $\log$ scale.

total detection rate of $97 \%$ of PSZ2 clusters with $z<0.2$. This percentage decreases to $93 \%$ when correlating our detections with all PSZ2 clusters.

Using the whole MCXC and PSZ2 catalogs and imposing our extended source criteria, out of the 1308 cluster candidates, 540 have a counterpart in MCXC, while 323 have a correspondence in PSZ2 (Fig. 4). These numbers overlap in 285 clusters, therefore we remain with 730 cluster candidates without counterparts in MCXC or PSZ2. The extent distribution of the cluster candidates is displayed in Fig. 4 . We find that $\sim 67 \%$ of our cluster candidates with extent $\lesssim 5$ arcmin have a counterpart in the MCXC or PSZ2 catalogs, showing the reliability of our extended source finder. The gray histogram in Fig. 4 shows the distribution of the cluster candidates with no counterpart in MCXC or PSZ2. Approximately $60 \%$ of these candidates have an extent $\gtrsim 5$ arcmin, leaving very extended sources as possible candidates for missed clusters in the RASS-based cluster catalogs.

\subsection{Looking for new galaxy clusters and groups}

As mentioned previously, the purpose of this paper is not to describe a new cluster catalog obtained by reprocessing the RASS data, but to show a pilot sample of promising cluster and group discoveries.

We further investigate the cluster candidates that have not previously been detected in the MCXC or PSZ2 catalogs. We use a correlation radius of 5 arcmin to match our 730 cluster candidates with the First ROSAT all-sky survey source catalog (1RXS, which includes the ROSAT All-Sky Survey Bright Source Catalog - BSC - Voges et al. 1999 and the ROSAT All-Sky Survey Faint Source Catalog - FSC - Voges et al. 2000), and the Second ROSAT all-sky survey source catalog (2RXS; Boller et al. 2016). We find 219 candidates with no detections in $1 R X S$ or $2 \mathrm{RXS}$. The distribution of these cluster candidates is shown in the orange histogram of Fig. 4, which shows larger values of extent than the candidates matched in the 1RXS or 2RXS catalogs. We concentrate from now on only on these cluster candidates.

To further validate the 219 cluster candidates we perform an exhaustive visual inspection and literature search. For the visual inspection, we used the ROSAT photon images, and their counterparts in infrared, optical, and microwave bands $\left(2 \mathrm{MASS}^{5}\right.$,

\footnotetext{
5 https://www.ipac.caltech.edu/2mass
}

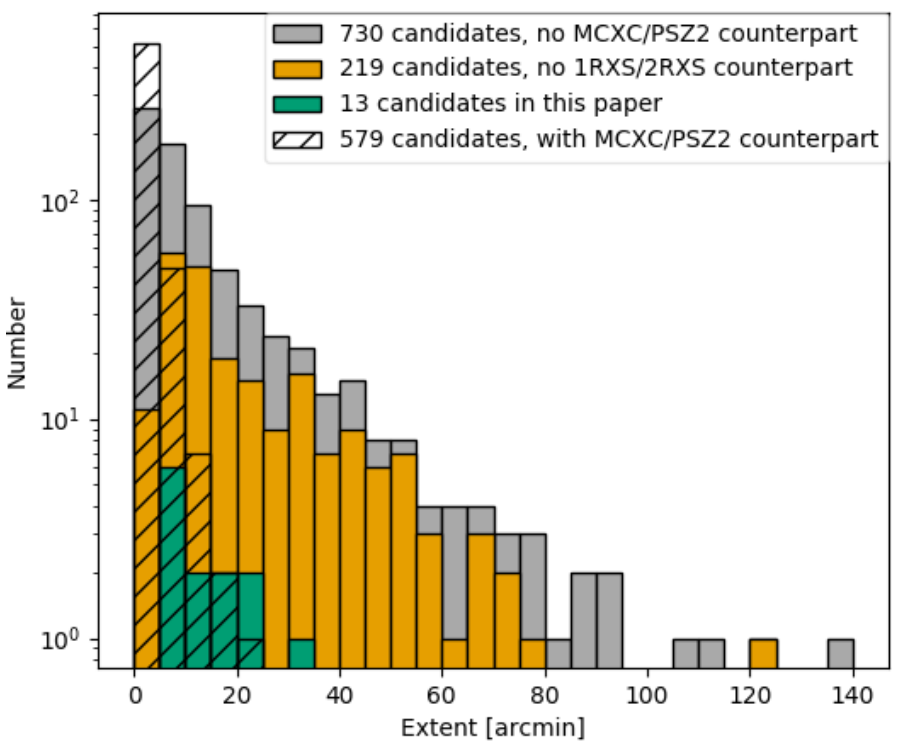

Fig. 4. Extent value distribution for the 1308 cluster candidates. Out of these 1308 candidates, 578 have a MCXC or PSZ2 counterpart (filled pattern histogram) and 730 do not have a counterpart in these catalogs (gray histogram). Out of the 730 candidates, 219 do not possess a counterpart in the 1RXS or 2RXS catalogs (orange histogram). The green histogram shows the extent distribution of the 13 group candidates discussed in detail in this work (see Sect. 4.3). The $y$-axis is a log scale.

$\mathrm{SDSS}^{6}, \mathrm{DSS}^{7}, \mathrm{ATLAS}^{8}$ and Planck ${ }^{9}$ ) overlaid with X-ray contours obtained from the wavelet-filtered images. The ROSAT exposure maps are also checked to make sure that the most extended detections are not artificially created by large exposure gradients. We also use images of the HI $4 \pi$ survey (HI4PI; HI4PI Collaboration 2016) to ensure that the cluster candidates are also not due to inhomogeneities in the HI sky distribution. We checked whether or not our cluster candidates have already been discovered by means of other X-ray, optical, or SZ surveys, and whether our source candidates are nearby galaxies given their extension. Table 2 shows the catalogs used to cross-check our candidates. Furthermore, we include spectroscopic redshift distributions obtained from SDSS DR $14^{10}$ or the NASA Extragalactic Database ${ }^{11}$ (NED) to assess the reliability of the cluster candidates. We do not restrict the redshift values when constructing these distributions.

Using the above information we subjectively rated each of the 219 cluster candidates with different grades: 1 for cluster candidates that are already known from the cluster catalogs shown in Table 2, 2 for cluster candidates that show a clear galaxy over-density in the infrared and/or optical images, 3 for cluster candidates for which it is not clear that a galaxy over-density exists due to lack of optical and/or infrared data, and 4 for cluster candidates that we consider as noise; for example, X-ray detections created by strong gradients of exposure in the RASS data or by low density value of $\mathrm{HI}$ in small regions of the sky. Our final grades show that $20 \%$ of the 219 cluster candidates are rated with $1,16 \%$ with $2,20 \%$ with 3 , and $44 \%$ with 4 . The

\footnotetext{
6 http://www.sdss.org

7 http://archive.eso.org/dss/dss

8 http://astro.dur.ac.uk/Cosmology/vstatlas

9 http://www.esa.int/Our\$_\$Activities/Space\$_\$Science/ Planck

10 http : //www . sdss . org/dr14/

11 https://ned.ipac.caltech.edu/
} 
number of cluster candidates rated as noise corresponds to 0.1 false extended detections per image. This rate is consistent with the number of misclassified AGNs and false extended sources per image in our simulations (see Sect. 3.3).

In this paper, we present a detailed analysis of a pilot sample of 13 cluster candidates selected out of those rated as grade 2. In optical, these cluster candidates coincide with clear overdensities of bright elliptical galaxies (see Fig. A.1) distributed over extended areas ( $\gtrsim 30$ arcmin) and NED shows that 11 out of the 13 cluster candidates have previously been reported in optical group and cluster catalogs. Furthermore, for all the candidates we are able to determine a reliable redshift estimation from existing galaxy catalogs (see Sect. 4.3.1). In the following section, we describe the methods used to characterize each individual cluster.

\subsection{Pilot sample of cluster candidates}

In this section, we describe the methods used to characterize the properties of 13 cluster candidates. Table 3 shows the sky coordinates of each system. The locations of these candidates in the extension likelihood - extent plane are shown with the black star symbols in the right-hand panel of Fig. 1.

\subsubsection{Redshift estimation}

We use NED to determine the redshift of each cluster candidate. We select all galaxies with spectroscopic redshift and within a box of size $1 \times 1 \mathrm{deg}^{2}$ centered on the cluster candidate position. For most of the cluster candidates, there are no or very few galaxies with redshift above 0.1 . The final redshift depends on the number of available redshifts and their distribution. We fit iteratively a Gaussian function to the redshift distribution in $z<0.1$ range, and apply $3 \sigma$ clipping to remove outliers. The redshift of candidate is derived from its peak (see right-hand panels Fig. A.1). For the cases with two peaks in the redshift distribution, we choose the closest redshift peak to the redshifts of the optical groups/clusters found in NED. Table 3 displays the total number of galaxies used for the redshift determination and the right-hand panels of Fig. A.1 shows their redshift distribution for each cluster candidate. We find that our 13 cluster candidates are in the redshift range $0.013-0.072$, with a median value of 0.036 . 11 of them are below $z<0.05$.

According to NED, 11 out of our 13 group candidates have at least one published counterpart within 15 arcmin (see Sect. 5 for details). We look into these catalogs to corroborate our redshift estimation. The red lines in the right-hand panels of Fig. A.1 represent the redshifts of these matched groups or clusters showing a good agreement with our estimation.

\subsubsection{Flux, luminosity, and mass determination}

Finally, we ran a more involved photometric analysis using the growth curve analysis method (hereafter GCA; Böhringer et al. 2000, 2001), which computes the ROSAT hard band, $0.5-2 \mathrm{keV}$, integrated count rates as a function of aperture around the estimated cluster center. For this, a local background is first estimated in a large annulus, then the background subtracted count rates is estimated at lower radii in bins of $0.5^{\prime}$ and summed within increasing apertures. Contamination by surrounding sources is corrected for by a deblending procedure that separates the signal in a number of angular sectors and excludes those falling outside $2.3 \sigma$ above/below the median value. The
Table 3. Galaxy groups in this pilot study.

\begin{tabular}{|c|c|c|c|c|}
\hline $\begin{array}{c}\text { Group } \\
\text { No. }\end{array}$ & $\begin{array}{c}\text { RA } \\
\text { J2000 }\end{array}$ & $\begin{array}{c}\text { Dec } \\
\text { J2000 }\end{array}$ & $z$ & $N_{\mathrm{g}}$ \\
\hline 1 & 215659.76 & +280057.6 & 0.069 & 6 \\
\hline 2 & 030402.64 & -120422.8 & 0.013 & 10 \\
\hline 3 & 105021.60 & +00 1644.4 & 0.039 & 42 \\
\hline 4 & 010519.44 & +360050.4 & 0.072 & 7 \\
\hline 5 & 022557.12 & +365945.6 & 0.036 & 17 \\
\hline 6 & 013651.36 & -140025.2 & 0.040 & 14 \\
\hline 7 & 231725.68 & +290231.2 & 0.023 & 8 \\
\hline 8 & 160624.00 & +154012.0 & 0.040 & 123 \\
\hline 9 & 165022.32 & +233542.0 & 0.036 & 52 \\
\hline 10 & 134918.96 & -071348.0 & 0.025 & 22 \\
\hline 11 & 210718.24 & -471008.4 & 0.017 & 10 \\
\hline 12 & 132856.88 & -280227.6 & 0.033 & 38 \\
\hline 13 & 024101.92 & +084655.2 & 0.021 & 14 \\
\hline
\end{tabular}

Notes. The sample is sorted according to the extent value of each group. Then, each group is identified using a sequential number (col. 1) to simplify their discussion in this paper. $N_{\mathrm{g}}$ is the number of galaxies used to estimate the redshift of candidates.

error on aperture count rates relies on Poisson statistics and is combined with the uncertainty on the background count rate. By default, the GCA method over ROSAT data uses a background annulus of 20-41.3', but given the large size of our cluster candidates, we had to increase these values manually for most sources, reaching up to $85-110^{\prime}$ for the most extended source.

At this point, we obtain a full radial profile of the source and a decision is needed as to the aperture within which the source flux should be measured. We closely follow the methods and assumptions laid out in Böhringer et al. (2013) for the REFLEXII cluster sample so that our flux measurements are comparable to this reference catalog. At first, a significance radius, $r_{\text {sig }}$, is determined for which the $1 \sigma$ uncertainty interval encompasses all count rates integrated in larger apertures. Then, the count rate within this aperture, $C R_{\text {sig }}$, is estimated by fitting a constant value to the plateau outside $r_{\text {sig. }}$. Since this aperture holds no physical meaning for the general cluster population, we convert it to a more meaningful value within the fixed spherical overdensity $r_{500}$ based on a few assumptions on the appearance of galaxy clusters.

For a given galaxy cluster mass, $M_{500}$, the integrated $0.1-2.4 \mathrm{keV}$ luminosity, $L_{500}$, and average spectroscopic temperature, $T_{\mathrm{X}}$, are estimated from the Reichert et al. (2011) scaling relations. The corresponding flux and count rates within $r_{500}$, which we refer to as $F_{500}$ and $C R_{500}$, are derived from the ROSAT PSPC response using appropriate corrections for spectral redshifting, flux dilution, and absorption. Then a $\beta$ model with $\beta=2 / 3$ and core radius $r_{\mathrm{c}}=r_{500} / 7$ is assumed to convert $C R_{500}$ to an expected count rates in the significance radius. This mapping enables us to iterate over cluster mass until the right count rate is obtained within $r_{\text {sig. }}$. This provides us with a set of estimated cluster parameters derived from the model $\left(r_{500}, F_{500}\right.$, $T_{\mathrm{X}}, L_{500}$, and $\left.M_{500}\right)$.

The third panel of each row in Fig. A.1 shows the growth curves estimated following the REFLEX-II procedure described above. As clearly seen from these plots, the observed growth curves (solid lines) are much flatter than the surface-brightness distribution predicted by the assumed $\beta$ model (dotted lines). This confirms that our detection algorithm permits us to identify very extended low-surface-brightness sources that may be 


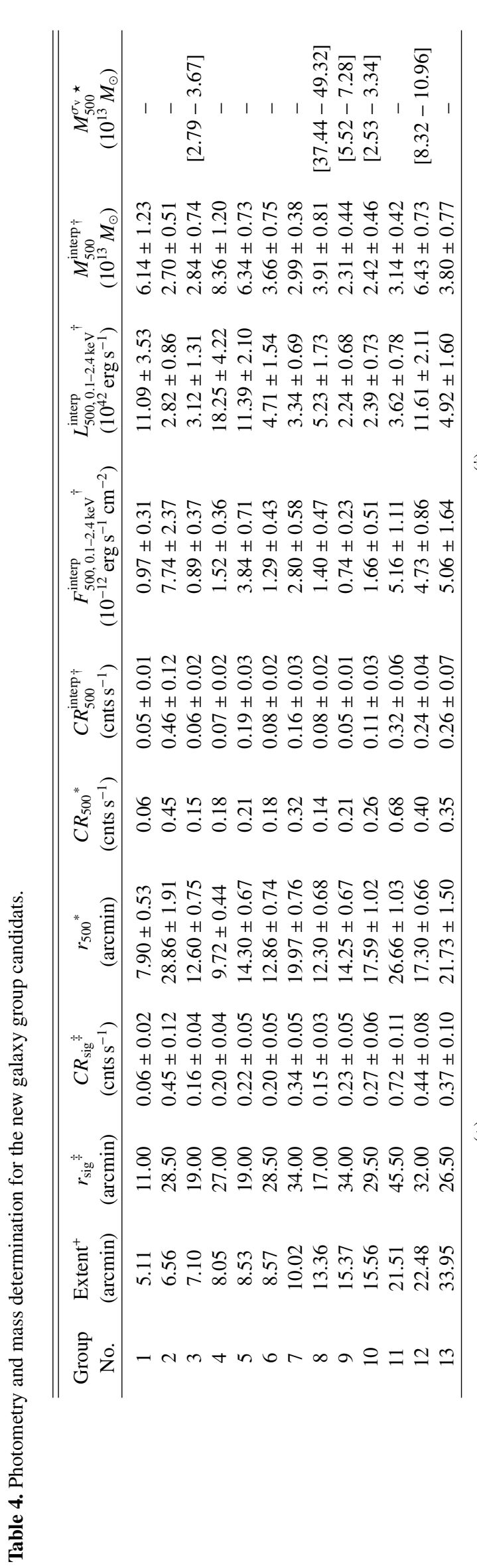

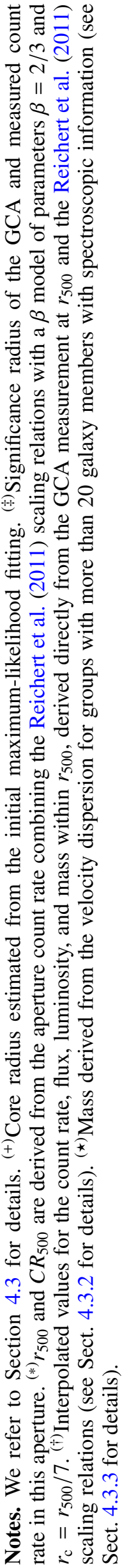

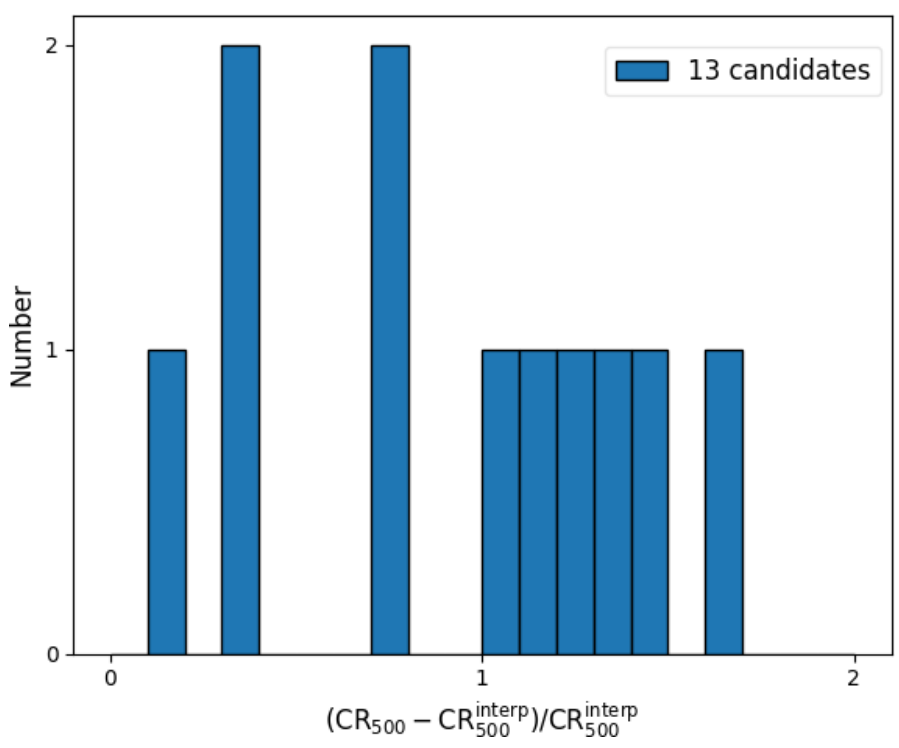

Fig. 5. Distribution of the normalized difference between $C R_{500}$ and $C R_{500}^{\text {interp }}$ (see Sect. 4.3.2 for details on the estimation of these quantities).

missing from previous catalogs. A downside, however, is that the assumed cluster profiles are not suitable to reliably convert fluxes from one radius to the other. Fortunately, the significance radius of the cluster emission exceeds the estimated value of $r_{500}$ for all of our detections. This permits to by-pass the use of a fixed surface-brightness model by comparing directly the count rates predicted by our scaling relation model for a given $M_{500}$ with the observed value within the corresponding $r_{500}$. A revised estimate of the count rate, $C R_{500}^{\text {interp }}$, is thus obtained by interpolating over the growth curve such that $C R\left(r_{500}\right) / C R_{500}\left(M_{500}\right)=1$. We note, however, that this new estimate of $C R_{500}^{\text {interp }}$ still includes a correction based on the default $\beta$ model, since we use it to estimate the dilution resulting from the ROSAT PSF, but it only makes a tiny difference for such extended systems. In addition, given the peculiar properties of our sources, they may not follow the average scaling relations used in the model. Consequently, although our quoted masses and fluxes always rely on the interpolated measurements, we preferred to list in Table 4 both values of $C R_{500}$ and $C R_{500}^{\text {interp }}$ as an illustration of systematic uncertainties. The differences between the two estimates are strongly variable (see Fig. 5), but the values of $C R_{500}$ are always overestimated by a factor of 2.05 on average.

The main characteristics of this pilot sample can be summarized as follows. The 13 cluster candidates have $r_{500} \gtrsim 8$ arcmin, reaching values up to $\sim 30 \mathrm{arcmin}$. Five of the 13 systems have flux measurements larger than the flux limit values of previous RASS cluster catalogs $\left(\gtrsim 3 \times 10^{-12} \mathrm{erg} \mathrm{s}^{-1} \mathrm{~cm}^{-2}\right.$, see Sect. 1). All 13 cluster candidates have $M_{500} \lesssim 10^{14} \mathrm{M}_{\odot}$, placing them in the group regime, rather than in the cluster scale. Therefore, from now on we refer to our 13 cluster candidates as galaxy groups.

\subsubsection{Dynamical mass estimation}

For the galaxy groups with more than 20 galaxy members with spectroscopic information (groups 3, 8, 9, 10, 12), we also calculated dynamical masses from their velocity dispersion. First, the velocity dispersion is obtained from the fit of the Gaussian function with a $3 \sigma$ clipping to the redshift distribution. Then, using Eq. 1 from Munari et al. (2013), which provides the relation between velocity dispersion and $M_{200}$, a range estimation of 


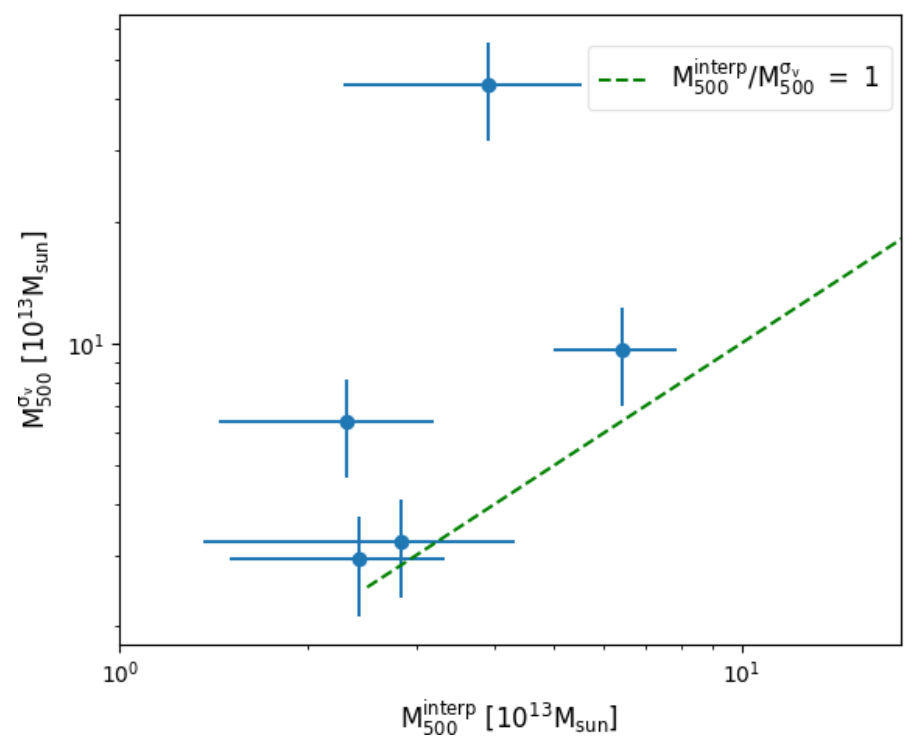

Fig. 6. Comparison of the derived dynamical masses with the masses derived from the $L_{\mathrm{X}}-M$ relation (see Sect. 4.3.3 for details).

$M_{200}^{\sigma_{v}}$ is derived. We further assume a Navarro-Frenk and White (NFW; Navarro et al. 1997) profile with concentration parameter $c=r_{200} / r_{\mathrm{s}}=4$ to determine a range of $M_{500}^{\sigma_{v}}$ by using $r_{500} \approx 0.65 r_{200}$ (Reiprich et al. 2013). The last column of Table 4 shows the range of values of the dynamical mass estimations. Figure 6 shows that the dynamical masses are in general agreement with the masses derived from the $L_{\mathrm{X}}-M$ relation (see Sect. 4.3.2). Only group 8 (see Table 3 ) shows a significant difference between these two mass estimations; this is further discussed in Sect. 5.8.

\section{Notes on individual groups}

In this paper, we focus on 13 new X-ray-selected galaxy groups that show very extended $\left(r_{\text {sig }}>10\right.$ arcmin $)$ emission in the RASS. Here we discuss each of them in detail.

Figure A.1 shows each of these systems through the RASS photon images and optical images from ATLAS, SDSS, or DSS, depending on the availability, and in that order of importance. These images have $1 \times 1 \mathrm{deg}^{2}$ size, except for the ATLAS images, which are $0.30 \times 0.30 \mathrm{deg}^{2}$. The red cross shows the determined position of the detected group from the maximum-likelihood fitting method, while the magenta plus symbols show the positions of X-ray sources in the 1RXS and 2RXS catalogs. The green contours are obtained from the respective wavelet-filtered images, and the blue circle shows the core radius determined from the maximum-likelihood fitting method (see Sect. 2). The cyan diamonds represent the positions of the individual galaxies with spectroscopic redshift information in NED and are used to determine the final redshift of the group (see Sect. 4.3.1).

As previously explained, the right-hand panels of Fig. A.1 show the redshift determination and Table 4 shows a summary of the parameters estimated for the 13 systems (see Sect. 4.2). We refer the reader to the right-hand panels of Fig. A.1 and Table 3 while reading the notes on the individual groups.

\subsection{Group 1}

NASA/IPAC Extragalactic Database provides redshift information for only six galaxies in this group at $z \sim 0.07$. The SDSS image (see Fig. A.1a) shows two clear and prominent brightest cluster galaxies (BCGs) at the center of our X-ray detection, for which there is no spectroscopic information in NED. From SDSS DR14, we found photometric redshifts of 0.059 and 0.068 for these central galaxies, which are consistent with our redshift estimation for this group $(z=0.069)$. This system is the most compact group in our pilot sample $\left(r_{500} \sim 8\right.$ arcmin $)$.

\subsection{Group 2}

According to NED, group 2 was previously identified as a galaxy group. Within 10 arcmin of the group position, two of these previous detections are: USGC s110, located 2.17 arcmin from the group center at $z=0.012$ (Ramella et al. 2002), and source 425 of Díaz-Giménez \& Zandivarez 2015, positioned $\sim 8.37$ arcmin away at $z=0.013$. These redshifts are consistent with our estimation for this group $(z=0.013$ from 10 galaxies). The ATLAS image shows two main dominating galaxies at the center of the group (see Fig. A.1b). This object is the most extended $\left(r_{500} \sim 30\right.$ arcmin $)$ and nearest group in our pilot sample. It has the largest flux of all groups in the sample $\left(F_{500}^{\text {interp }} \sim\right.$ $7.74 \times 10^{-12} \mathrm{erg} \mathrm{s}^{-1} \mathrm{~cm}^{-2}$ ), which is 2.6 times larger than the flux limit of the REFLEX catalog $\left(3 \times 10^{-12} \mathrm{erg} \mathrm{s}^{-1} \mathrm{~cm}^{-2}\right.$ in the 0.1-2.4 keV energy band, Böhringer et al. 2001).

\subsection{Group 3}

This group has previously been identified in several publications; for example, as SDSS-C4 1156 at $z=0.039$ by von der Linden et al. (2007), and as PM2GC GR1204 at $z=$ 0.040 by Calvi et al. (2011). These works provided several spectroscopic redshifts (for 42 galaxies), which allow us to obtain a redshift estimation of $z=0.039$. Moreover, this group has several elliptical galaxies easily spotted by eye (see Fig. A.1c).

\subsection{Group 4}

Group 4 presents a similar situation to group 1 (see Sect. 5.1). NED provides redshift information for only seven galaxies, obtaining an estimated redshift $z=0.072$ for this system. From the 2MASS Photometric Redshift catalog (2MPZ; Bilicki et al. 2014), we found photometric redshifts of 22 galaxies. These redshift values are consistent with the final redshift estimation of this galaxy group. The spatial distribution of these galaxies coincides with the X-ray contours of this system. This group has the highest redshift value in our pilot sample. Although this object is located near a bright point-like source and surrounded by another two weak RXS point-like sources, our detection algorithm is capable of disentangling all this information and still finds this extended source. The DSS image shows a bright galaxy near the X-ray peak emission of the group (see Fig. A.1d). This system is the most massive $\left(M_{500}^{\text {interp }} \sim 8.36 \times 10^{13} M_{\odot}\right)$ and most luminous $\left(L_{500}^{\text {interp }} \sim 18.25 \times 10^{42} \mathrm{erg} \mathrm{s}^{-1}\right)$ group in our pilot sample.

\subsection{Group 5}

Similar to group 2 (see Sect. 5.2), this system has been identified in different works: Colless et al. (2001; identified as CID 15 at $z=0.036$ and located 5.04 arcmin away from the group center), and Ramella et al. (2002; labeled as USGC U118 at $z=0.035$ and located 8.64 arcmin away). Both optical detections have 
similar redshifts to the one determined from 17 galaxy members $(z=0.036)$. The DSS image (see Fig. A.1e) clearly shows elliptical galaxies in this field.

\subsection{Group 6}

This group has also been identified as a galaxy triplet system, APMUKS(BJ) B013430.06-14130 (Maddox et al. 1990), located 2.89 arcmin away from the group center, as a galaxy group USGC s061 at $z=0.039,6.70 \mathrm{arcmin}$ away (Ramella et al. 2002), and as a galaxy group named [DZ2015] 396 at $z=0.039$ located 7.26 arcmin away (Díaz-Giménez \& Zandivarez 2015). Although NED only provides redshifts for 14 galaxies, the estimated redshift $(z=0.040)$ of the group is consistent with the ones of the identified close groups. The ATLAS image (Fig. A.1f) clearly shows a dominant galaxy in the system with $z=0.040$ (Huchra et al. 1993).

\subsection{Group 7}

In a similar situation as group 5 (see Sect. 5.5), NED shows an identified optical cluster counterpart 1.92 arcmin away from this group. This matching group is called WBL 704 at $z=0.024$ (White et al. 1999). This redshift is consistent with our estimation for this group ( $z=0.023$ from 8 galaxies). The SDSS image shows a dominant BCG (see Fig. A.1g).

\subsection{Group 8}

For this system, there are 123 galaxies with redshifts measured from NED, giving $z=0.040$ as the final result. It has been previously reported by Smith et al. (2012) as MSPM 00022 located 1.94 arcmin away from the group center at $z=0.039$, which is consistent with our redshift determination. The SDSS image shows many elliptical galaxies gathering around a prominent BCG (Fig. A.1h). This group is located 47.96 arcmin away from cluster Abell 2152 at $z=0.041$ (Struble \& Rood 1999) with $r_{500}=12.99 \operatorname{arcmin}$ (Piffaretti et al. 2011). Given that our group presents a similar redshift, we might consider it as a potential infalling group into Abell 2152. This might be reflected by the large discrepancy between its $M_{500}^{\text {interp }}\left(\sim 3.91 \times 10^{13} M_{\odot}\right)$ and $M_{500}^{\sigma_{v}}\left(\sim 37.44 \times 10^{13} M_{\odot}\right)$ values. However, further investigation is needed.

\subsection{Group 9}

Similar to group 5 (see Sect. 5.5), this system has an optical counterpart, MSPM 00069 (Smith et al. 2012), located 5.50 arcmin away at $z=0.035$, which is consistent with our redshift estimation for this group ( $z=0.036$ from 52 galaxies). ROSAT catalogs and images (Fig. A.1i) show that this group is surrounded by point-like sources, but our detection algorithm is able to disentangle the X-ray emission and detect them individually. This group is the least massive $\left(M_{500}^{\text {interp }} \sim 2.31 \times 10^{13} M_{\odot}\right)$, the least luminous $\left(L_{500}^{\text {interp }} \sim 2.24 \times 10^{42} \mathrm{erg} \mathrm{s}^{-1}\right)$ and has the smallest flux $\left(F_{500}^{\text {interp }} \sim 0.74 \times 10^{-12} \mathrm{erg} \mathrm{s}^{-1} \mathrm{~cm}^{-2}\right)$ of all groups in the sample.

\subsection{Group 10}

This system has been reported in previous works as HCG 067 (Hickson 1982) and as LDCE 1002 (Crook et al. 2008), at redshifts of 0.025 and 0.024 , respectively. These redshifts are consistent with the one we have estimated for this group $(z=$ 0.025 from 22 galaxies). Although this group is surrounded by three bright RXS sources (Fig. A.1j), our detection algorithm is able to disentangle the individual emission. The ATLAS image clearly shows several elliptical galaxies in this system.

\subsection{Group 11}

Within 10 arcmin of the position of this group, we found two Abell clusters: Abell S0924 at $z=0.016$ and Abell 3742 at $z=0.016$ (Abell et al. 1989). These redshifts are fairly consistent with our redshift estimation of $z=0.017$ for this group, which was obtained from ten galaxies. Given that this group displays two prominent BCGs (Fig. A.1k), it has been identified in other works as a galaxy group at a redshift similar to our estimation (e.g., Chow-Martínez et al. 2014a).

\subsection{Group 12}

From 38 galaxy redshifts, we determined $z=0.033$ for this system. The DSS image (see Fig. A.11) shows several elliptical galaxies in the area of this group. It coincides with several known optical counterparts. The most representative are groups 266 of Díaz-Giménez \& Zandivarez (2015) and 77 of Ragone et al. (2006), both with a redshift of $z=0.034$ and located 5.44 and $\sim 8$ arcmin from our detection, respectively. In a similar situation as for group 8 , it is located only 27.00 arcmin away from galaxy cluster Abell S0736, also at $z=0.033$ (Chow-Martínez et al. 2014b), and 58.95 arcmin from galaxy cluster Abell 1736 at $z=0.046$ (Planck Collaboration XXVII 2016a). This group is actually a member of the Front-Eastern-Wall of the Shapley supercluster. According to the groups identified in the area by Ragone et al. (2006), it most likely resides in a filamentary structure starting from Abell S0736 $(z=0.033)$, going through our source $(z=0.033)$, then Abell $1736 \operatorname{NED01}(z=0.035)$, Abell 1736 NED02 $(z=0.041)$, and ending at Abell 1736 $(z=0.0453)$, all nicely aligned also in the plane of the sky. This is further supported by their location in the outskirts of the Shapley supercluster (they have some of the lowest redshifts among the identified supercluster members).

\subsection{Group 13}

Based on 14 redshifts found in NED, we determine $z=0.021$ for this group. It has three optical counterparts at similar redshift within 5 arcmin: galaxy pair NGC 1044 (Falco et al. 1999), galaxy triple HDCE 0161 (Crook et al. 2008), and galaxy group USGC U142 (Ramella et al. 2002). Croston et al. (2008) observed NGC 1044 with XMM-Newton to follow up an extended radio source, detecting extended X-ray emission from a group environment. The DSS image (see Fig. A.1m) shows a galaxy pair dominating the system.

\section{Discussion}

The 13 groups in our pilot sample have extent values (i.e., core radius) larger than 5 arcmin. Of these 13 groups, 11 have a redshift lower than 0.05 . For all of them, significant emission can be traced out to $r_{\text {sig }}>10$ arcmin (see Table 3). These facts show that indeed some local, very extended galaxy groups have been missed in previous works and that our methodology used to look for them works fine. 
The flux measurements of our groups show that these 13 systems are above the flux limit of the 1RXS and 2RXS cata$\operatorname{logs}\left(\sim 10^{-13} \mathrm{erg} \mathrm{s}^{-1} \mathrm{~cm}^{-2}\right.$ for $2 \mathrm{RXS}$ catalog, Boller et al. 2016). Since most RASS galaxy cluster catalogs rely on $1 \mathrm{RXS} / 2 \mathrm{RXS}$ as initial source sample, it is not surprising that the galaxy groups are also not included in any published RASS cluster catalog, despite their fluxes often being larger than the flux limits of those.

Our growth curve analysis method shows that our groups have a relatively flat $(\beta<2 / 3)$ surface-brightness distribution (see the third panel of each row in Fig. A.1), making this pilot sample a perfect representation of sources that do not have the expected or usual characteristics and therefore are missed in catalogs. This is supported by the locations of the 13 groups in the extension likelihood - extent plane (see right-hand panel of Fig. 1), which are consistent with detected clusters of low $\beta$ values in our simulations (low values of extension likelihood and large values of extent, see Fig. 2).

All these features make our 13 groups perfect candidates to be missed by the sliding-cell algorithm used to construct the RASS catalogs. This source finder performs best when detecting point-like sources (Rosati et al. 2002).

The fact that most of our secure group candidates lie below $z=0.05$ raises the question of whether there is a significant fraction of such systems that have been missed. Around $14 \%$ of the MCXC clusters, that is, 251, have a redshift no larger than $z=0.05$. A similar number, 219 objects, constitutes our sample of group candidates with no 1RXS/2RXS detections. If further analysis shows that at least $50 \%$ of these systems are indeed galaxy groups we will increase the number of known X-ray galaxy groups at $z<0.05$ by at least $40 \%$. Schellenberger \& Reiprich (2017) have shown the impact on the values of $\Omega_{\mathrm{m}}$ and $\sigma_{8}$ by the incompleteness of the mass function in the galaxy group regime. Generally, if there is an uncorrected incompleteness of $25 \%-50 \%$ at low masses, the best-fit $\Omega_{\mathrm{m}}$ values are biased low.

\section{Conclusions}

In this work, we have reprocessed the ROSAT All-Sky Survey (RASS) data with the aim to look for very extended, low-surfacebrightness galaxy groups and clusters that might have been missed in previous works. Our approach consisted of a waveletbased source-detection algorithm and a maximum-likelihood fitting method to characterize the source properties. We tested our methodology on extensive Monte-Carlo simulations and determined the selection criteria for significant and extended sources.

We found 1308 cluster candidates in the RASS data. Approximately $44 \%$ of those systems have a cluster counterpart in either MCXC or PSZ2 catalogs. This makes our methodology a reliable procedure. From the $\sim 56 \%$ remaining, $70 \%$ of them have a counterpart in either $1 \mathrm{RXS}$ or $2 \mathrm{RXS}$ catalogs. In this paper, we focus on and perform an extensive visual inspection and literature search of the other $30 \%$ of the systems. Of these objects, we describe in detail the analysis and characterization of a pilot sample of 13 candidates.

We determined a spectroscopic redshift for each cluster candidate finding that most of them are local systems $(z \lesssim 0.05)$, and are very extended (significant X-ray emission traced out to more than 10 arcmin radius). Moreover, all these objects have fluxes above the limit of the 1RXS and 2RXS catalogs. We argue that they might have been missed because of the source finder used in previous works, that is, the sliding-cell algorithm, which is not optimal for finding such low-flux, very extended objects. Our pilot sample also reveals that the surface-brightness distribution of the systems is flatter than the usual profile, that is $\beta<2 / 3$, and that the missed systems are located in the group regime given the determined masses from galaxy velocity dispersions or a $L_{\mathrm{X}}-M$ scaling relation $\left(M_{500} \lesssim 10^{14} M_{\odot}\right)$.

In a subsequent paper, we will present and discuss in detail the remaining cluster candidates that we have found.

Acknowledgements. The authors wish to thank Lijun Gou, Angus Wright, Jens Erler, Chaoli Zhang, Zhonglue Wen, and Jinlin Han for useful help, discussions and support during the development of this paper. This work was supported by the German Research Association (DFG) through the Transregional Collaborative Research Centre TRR33 The Dark Universe (project B18) and the German Aerospace Agency (DLR) with funds from the Ministry of Economy and Technology (BMWi) through grant 50 OR 1514. The authors thank the support from the CAS-DAAD Joint Fellowship Programme for Doctoral Students of Chinese Academy of Sciences (ST 34). This work is partially supported by the National Program on Key Research and Development Project (Grant No. 2016YFA0400800). WX acknowledges the support of the Chinese Academy of Sciences through grant No. XDB23040100 from the Strategic Priority Research Program and that of the National Natural Science Foundation of China with grant No. 11333005. This research has made use of the NASA/IPAC Extragalactic Database (NED), which is operated by the Jet Propulsion Laboratory, California Institute of Technology, under contract with the National Aeronautics and Space Administration.

\section{References}

Abbott, T., Abdalla, F. B., Allam, S., et al. 2016, Phys. Rev. D, 94, 022001

Abell, G. O., Corwin, Jr., H. G., \& Olowin, R. P. 1989, ApJS, 70, 1

Allen, S. W., Evrard, A. E., \& Mantz, A. B. 2011, ARA\&A, 49, 409

Bertin, E., \& Arnouts, S. 1996, A\&AS, 117, 393

Bilicki, M., Jarrett, T. H., Peacock, J. A., Cluver, M. E., \& Steward, L. 2014, ApJS, 210, 9

Boese, F. G. 2000, A\&AS, 141, 507

Böhringer, H., Voges, W., Huchra, J. P., et al. 2000, ApJS, 129, 435

Böhringer, H., Schuecker, P., Guzzo, L., et al. 2001, A\&A, 369, 826

Böhringer, H., Chon, G., Collins, C. A., et al. 2013, A\&A, 555, A30

Boller, T., Freyberg, M. J., Trümper, J., et al. 2016, A\&A, 588, A103

Borgani, S., Rosati, P., Tozzi, P., et al. 2001, ApJ, 561, 13

Calvi, R., Poggianti, B. M., \& Vulcani, B. 2011, MNRAS, 416, 727

Cash, W. 1979, ApJ, 228, 939

Cavaliere, A., \& Fusco-Femiano, R. 1976, A\&A, 49, 137

Chow-Martínez, M., Andernach, H., Caretta, C. A., \& Trejo-Alonso, J. J. 2014a, MNRAS, 445, 4073

Chow-Martínez, M., Andernach, H., Caretta, C. A., \& Trejo-Alonso, J. J. 2014b, MNRAS, 445, 4073

Colless, M., Saglia, R. P., Burstein, D., et al. 2001, MNRAS, 321, 277

Crook, A. C., Huchra, J. P., Martimbeau, N., et al. 2008, ApJ, 685, 1320

Croston, J. H., Hardcastle, M. J., Birkinshaw, M., Worrall, D. M., \& Laing, R. A. 2008, MNRAS, 386, 1709

de Haan, T., Benson, B. A., Bleem, L. E., et al. 2016, ApJ, 832, 95

Díaz-Giménez, E., \& Zandivarez, A. 2015, A\&A, 578, A61

Dunkley, J., Komatsu, E., Nolta, M. R., et al. 2009, ApJS, 180, 306

Ebeling, H., Voges, W., Bohringer, H., et al. 1996, MNRAS, 281, 799

Ebeling, H., Edge, A. C., Bohringer, H., et al. 1998, MNRAS, 301, 881

Ebeling, H., Edge, A. C., Allen, S. W., et al. 2000, MNRAS, 318, 333

Faccioli, L., Pacaud, F., Sauvageot, J.-L., et al. 2018, A\&A, 620, A9

Falco, E. E., Kurtz, M. J., Geller, M. J., et al. 1999, PASP, 111, 438

Harnden, Jr., F. R., Fabricant, D. G., Harris, D. E., \& Schwarz, J. 1984, SAO Special Report, 393

Hasselfield, M., Hilton, M., Marriage, T. A., et al. 2013, J. Cosmology Astropart. Phys., 7, 008

Henry, J. P., Evrard, A. E., Hoekstra, H., Babul, A., \& Mahdavi, A. 2009, ApJ, 691, 1307

HI4PI Collaboration, Ben Bekhti, N., \& Flöer, L. ,et al. 2016, A\&A, 594, A116

Hickson, P. 1982, ApJ, 255, 382

Hildebrandt, H., Viola, M., Heymans, C., et al. 2017, MNRAS, 465, 1454

Hilton, M., Hasselfield, M., Sifón, C., et al. 2018, ApJS, 235, 20

Hinshaw, G., Larson, D., Komatsu, E., et al. 2013, ApJS, 208, 19

Huchra, J., Latham, D. W., da Costa, L. N., Pellegrini, P. S., \& Willmer, C. N. A. 1993, AJ, 105, 1637

Kowalski, M., Rubin, D., Aldering, G., et al. 2008, ApJ, 686, 749

Liu, T., Tozzi, P., Tundo, E., et al. 2015, ApJS, 216, 28 
Lloyd-Davies, E. J., Romer, A. K., Mehrtens, N., et al. 2011, MNRAS, 418, 14 Maddox, S. J., Efstathiou, G., Sutherland, W. J., \& Loveday, J. 1990, MNRAS 243, 692

Mantz, A., Allen, S. W., Rapetti, D., \& Ebeling, H. 2010, MNRAS, 406, 1759

Marriage, T. A., Acquaviva, V., Ade, P. A. R., et al. 2011, ApJ, 737, 61

McCarthy, I. G., Bird, S., Schaye, J., et al. 2018, MNRAS, 476, 2999

Mehrtens, N., Romer, A. K., Hilton, M., et al. 2012, MNRAS, 423, 1024

Moretti, A., Campana, S., Lazzati, D., \& Tagliaferri, G. 2003, ApJ, 588, 696

Munari, E., Biviano, A., Borgani, S., Murante, G., \& Fabjan, D. 2013, MNRAS, 430, 2638

Navarro, J. F., Frenk, C. S., \& White, S. D. M. 1997, ApJ, 490, 493

Oguri, M., Lin, Y.-T., Lin, S.-C., et al. 2018, PASJ, 70, S20

Pacaud, F., Pierre, M., Refregier, A., et al. 2006, MNRAS, 372, 578

Pacaud, F., Clerc, N., Giles, P. A., et al. 2016, A\&A, 592, A2

Piffaretti, R., Arnaud, M., Pratt, G. W., Pointecouteau, E., \& Melin, J.-B. 2011, A\&A, 534, A109

Planck Collaboration XX. 2014, A\&A, 571, A20

Planck Collaboration XXVII. 2016a, A\&A, 594, A27

Planck Collaboration XXIV. 2016b, A\&A, 594, A24

Press, W. H., Teukolsky, S. A., Vetterling, W. T., \& Flannery, B. P. 1992, Numerical recipes in $\mathrm{C}$. The art of scientific computing (Cambridge: University Press)

Ragone, C. J., Muriel, H., Proust, D., Reisenegger, A., \& Quintana, H. 2006, A\&A, 445, 819

Ramella, M., Geller, M. J., Pisani, A., \& da Costa, L. N. 2002, AJ, 123, 2976

Reichert, A., Böhringer, H., Fassbender, R., \& Mühlegger, M. 2011, A\&A, 535, A4

Reiprich, T. H., \& Böhringer, H. 2002, ApJ, 567, 716

Reiprich, T. H., Basu, K., Ettori, S., et al. 2013, Space Sci. Rev., 177, 195

Rosati, P., Della Ceca, R., Burg, R., Norman, C., \& Giacconi, R. 1995, ApJ, 445, L11
Rosati, P., Borgani, S., \& Norman, C. 2002, ARA\&A, 40, 539

Rykoff, E. S., Rozo, E., Busha, M. T., et al. 2014, ApJ, 785, 104

Rykoff, E. S., Rozo, E., Hollowood, D., et al. 2016, ApJS, 224, 1

Schellenberger, G., \& Reiprich, T. H. 2017, MNRAS, 471, 1370

Seljak, U. 2002, MNRAS, 337, 769

Sinnott, R. W. 1988, in NGC 2000.0: The Complete New General Catalogue and Index Catalogues of Nebulae and Star Clusters, ed. J. L. E. Dreyer (Cambridge: Sky Publishing Corporation and Cambridge University Press)

Smith, A. G., Hopkins, A. M., Hunstead, R. W., \& Pimbblet, K. A. 2012, MNRAS, 422, 25

Spergel, D. N., Verde, L., Peiris, H. V., et al. 2003, ApJS, 148, 175

Spergel, D. N., Bean, R., Doré, O., et al. 2007, ApJS, 170, 377

Starck, J.-L., \& Pierre, M. 1998, A\&AS, 128, 397

Struble, M. F., \& Rood, H. J. 1999, ApJS, 125, 35

Truemper, J. 1992, MNRAS, 33, 165

Truemper, J. 1993, Science, 260, 1769

Ueda, Y., Akiyama, M., Hasinger, G., Miyaji, T., \& Watson, M. G. 2014, ApJ, 786, 104

Valtchanov, I., Pierre, M., \& Gastaud, R. 2001, A\&A, 370, 689

Viana, P. T. P., Nichol, R. C., \& Liddle, A. R. 2002, ApJ, 569, L75

Vikhlinin, A., McNamara, B. R., Forman, W., et al. 1998, ApJ, 502, 558

Voges, W., Aschenbach, B., Boller, T., et al. 1999, A\&A, 349, 389

Voges, W., Aschenbach, B., \& Boller, T. 2000, IAU Circ., 7432

von der Linden, A., Best, P. N., Kauffmann, G., \& White, S. D. M. 2007, MNRAS, 379, 867

Wen, Z. L., Han, J. L., \& Liu, F. S. 2009, ApJS, 183, 197

Wen, Z. L., Han, J. L., \& Liu, F. S. 2012, ApJS, 199, 34

Wen, Z. L., Han, J. L., \& Yang, F. 2018, MNRAS, 475, 343

White, R. A., Bliton, M., Bhavsar, S. P., et al. 1999, AJ, 118, 2014

Zwicky, F., Kowal, C. T., et al. "Catalogue of Galaxies and of Clusters of Galaxies" Volume VI (Pasadena: California Institute of Technology) 


\section{Appendix A: Additional figure}
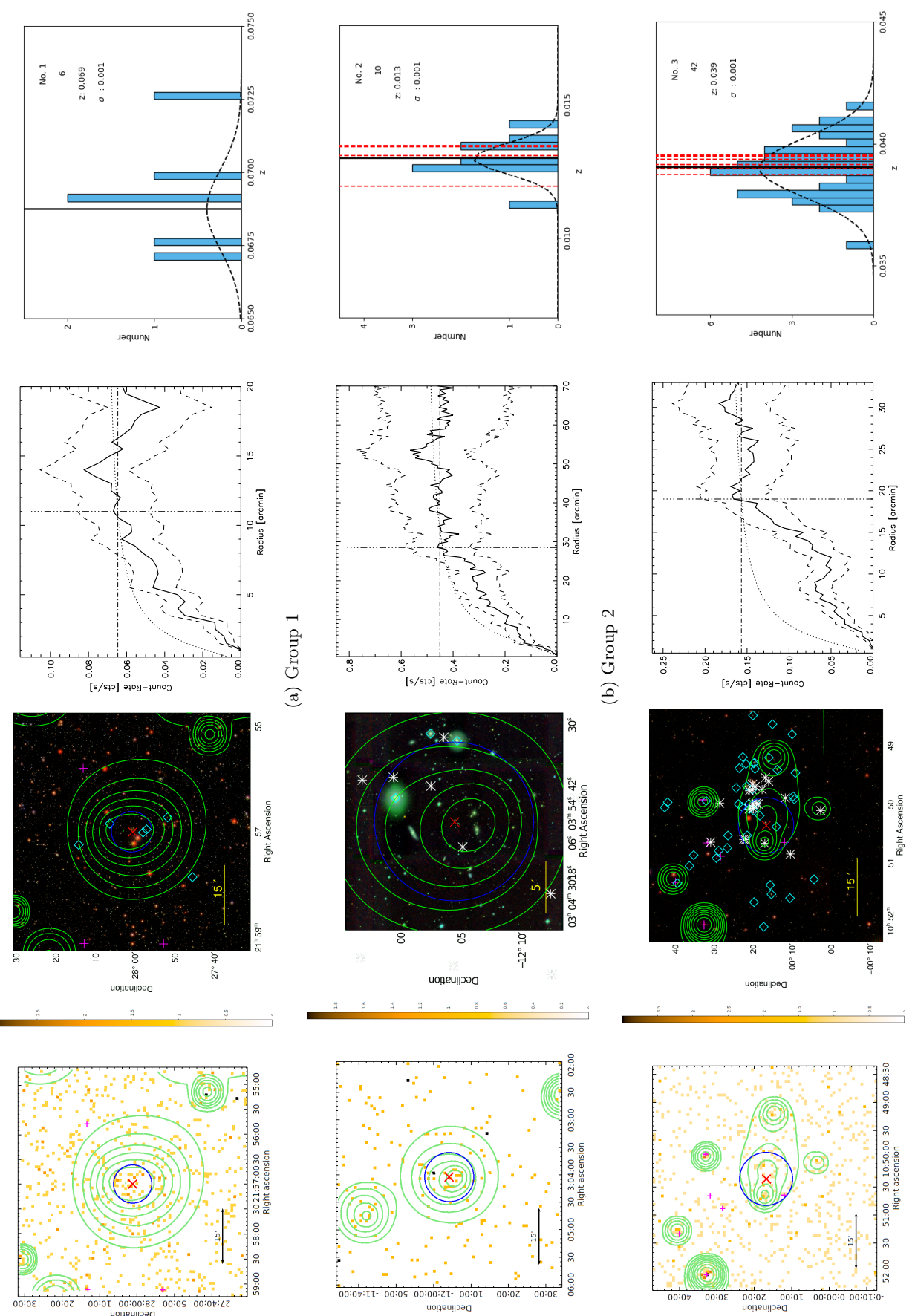

Fig. A.1. From left to right: RASS photon images (left), optical images, integrated count rates profiles, and spectroscopic redshift histograms (right) for the galaxy groups in our pilot sample. The optical images are from ATLAS (composed of $g, r, i$ bands, group 2, 6, 10), SDSS (composed of $g, r, i$ bands, group $1,3,7,8,9)$ or DSS ( $r$ band, other groups). The images have a $1 \times 1 \mathrm{deg}^{2}$ size, except for the ATLAS images, which are $0.30 \times 0.30 \mathrm{deg}^{2}$. The red cross shows the position of the group candidate. The magenta plus signs display the location of $1 \mathrm{RXS}$ or $2 \mathrm{RXS}$ sources. The cyan diamonds show the positions of galaxies used for redshift determination. The white asterisks show the positions of known optical galaxy groups/clusters obtained from NED. The green contours are obtained from the corresponding wavelet-filtered images, and the blue circle shows the extent value (i.e., the core radius) of the group. The integrated count rates profile is background subtracted (solid line). The dashed curves give the $1 \sigma$ statistical error of the count rates. The vertical and horizontal dot-dashed lines indicate the aperture radius and the count rates used for the primary photometric results. The dotted curve shows the $\beta$ model of the cluster modelling used to extrapolate to $r_{500}$ (see Sect. 4.3 .2 for further details). In the spectroscopic redshift histograms the redshift values are taken for all galaxies within a box of $1 \times 1 \mathrm{deg}^{2}$ centered on the group position with $z<0.10$ from NED. The total number of galaxies used in each redshift determination is given by the second number displayed in the upper right corner of each panel (see also Table 3). The redshift distribution is fitted with a Gaussian function using $3 \sigma$ clipping (black dashed curve). The final redshift of each group is taken from the peak of the fitting, and it is shown by the third number displayed in the upper-right corner of each panel and by the black solid line. The red lines show the redshifts of identified groups or clusters within 15 arcmin of the position of our candidates, red dashed line for spectroscopic redshift and the red dotted line for photometric redshift (see Sect. 5 for further details). 
A\&A 619, A162 (2018)
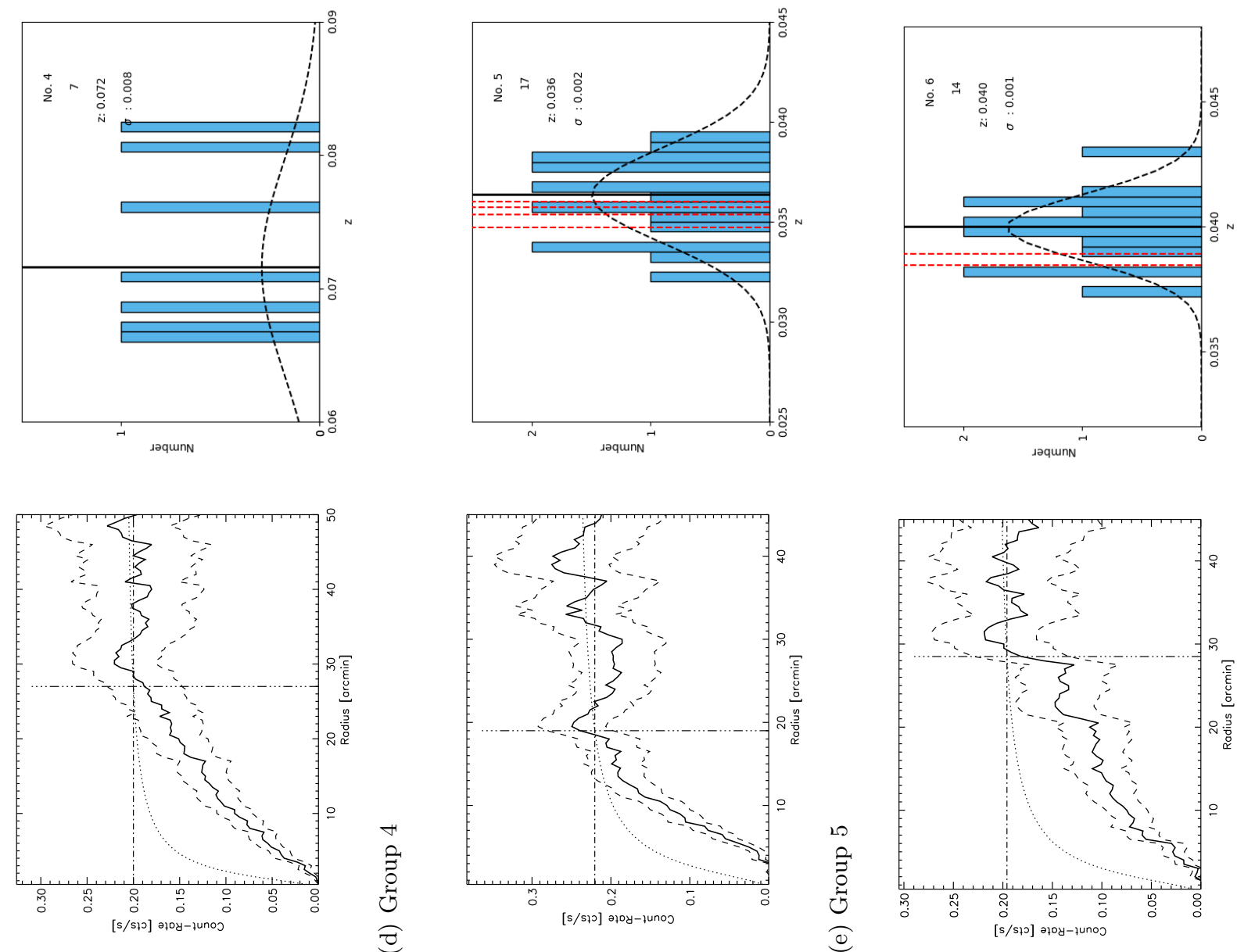

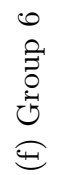
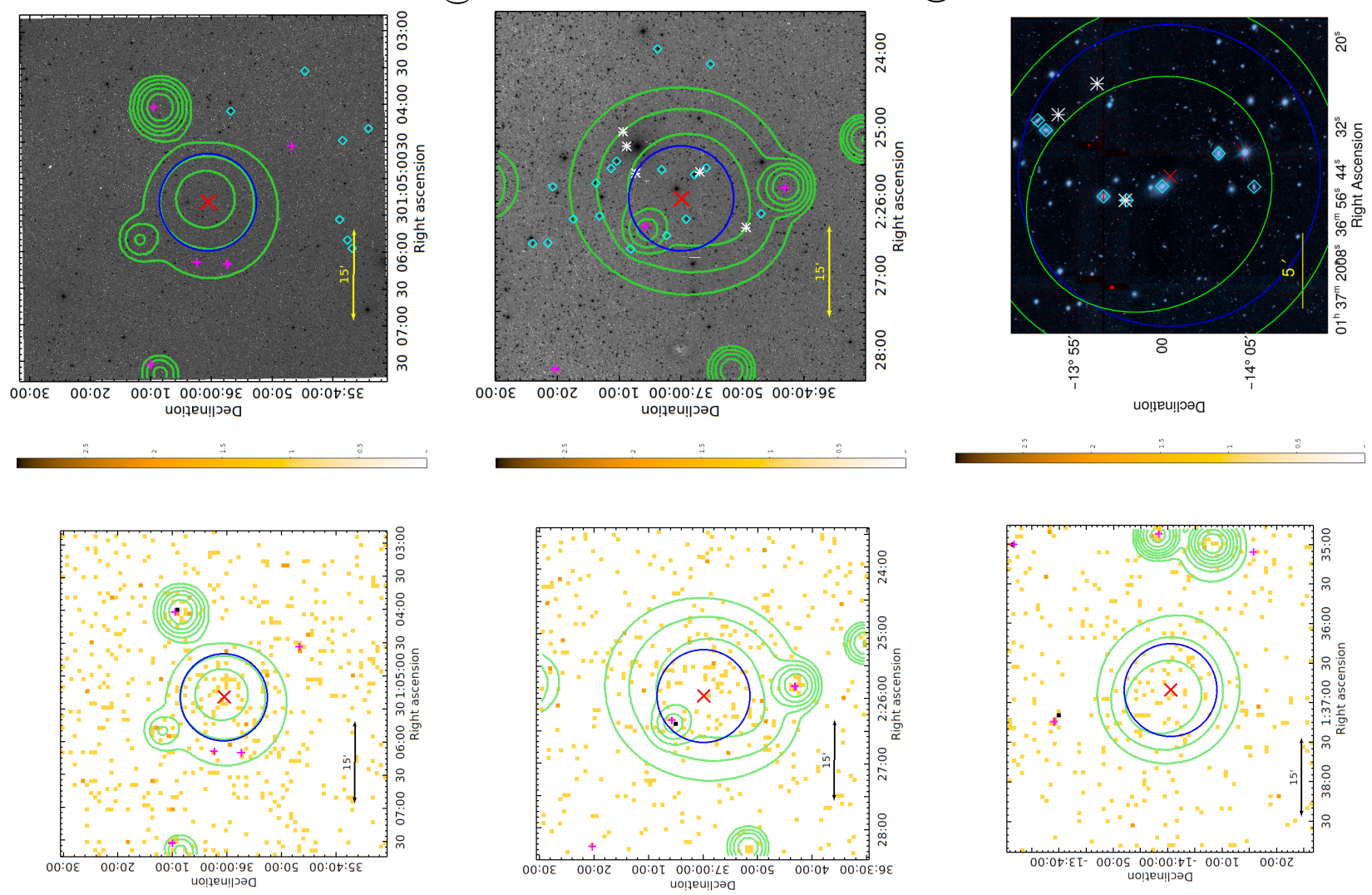

Fig. A.1. continued. 
W. Xu et al.: A sample of new extended galaxy groups from the RASS
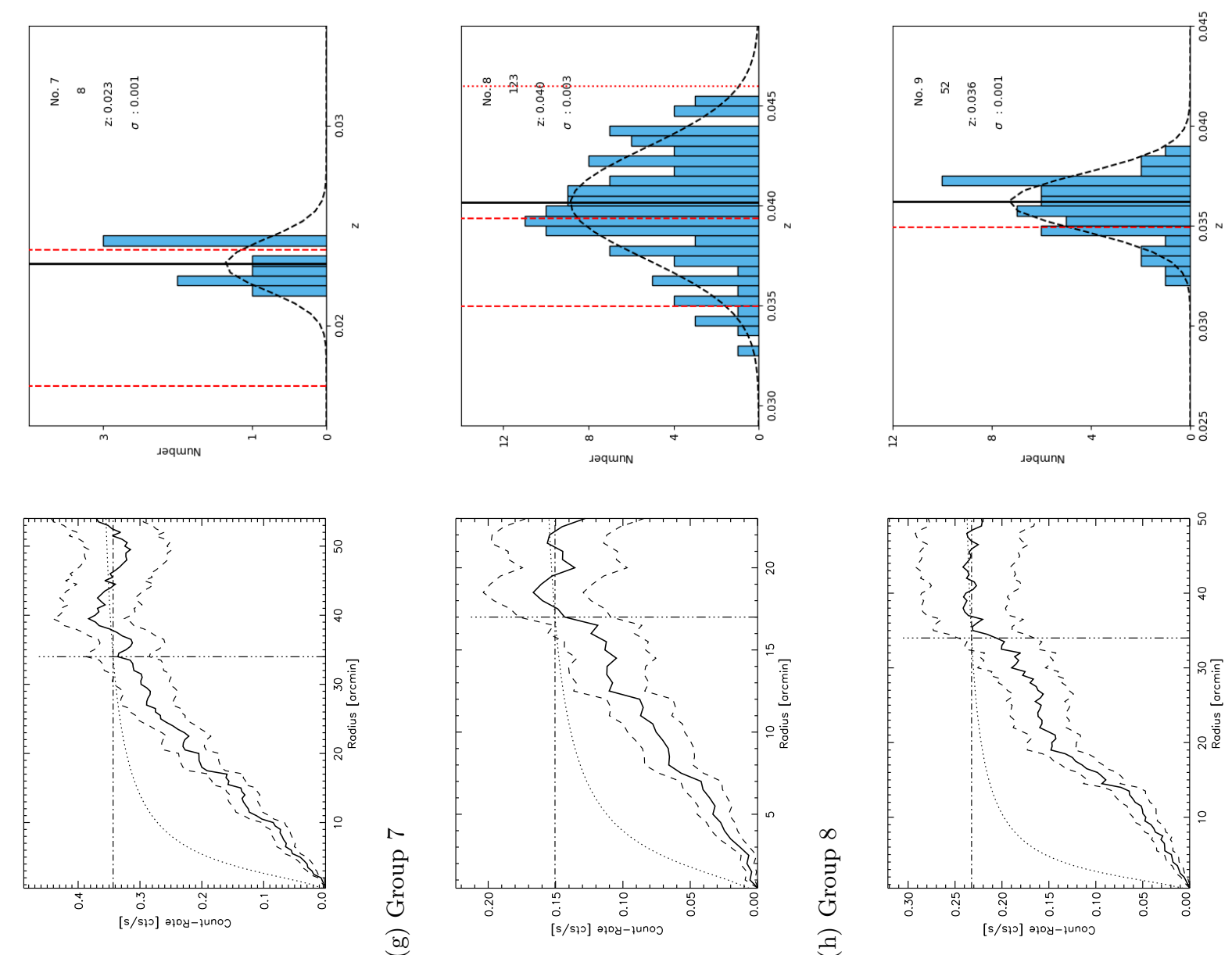

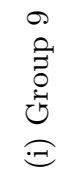
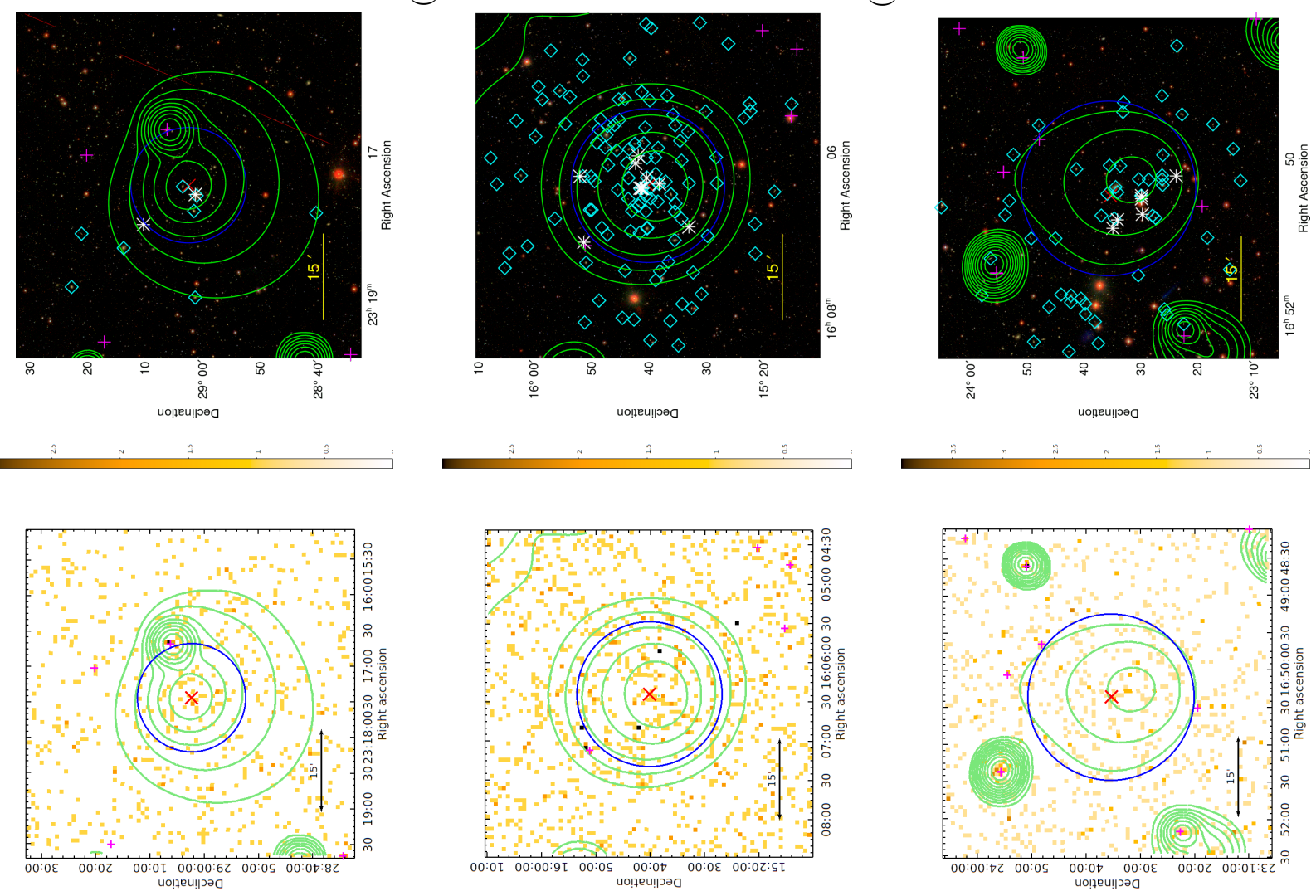

Fig. A.1. continued. 
A\&A 619, A162 (2018)
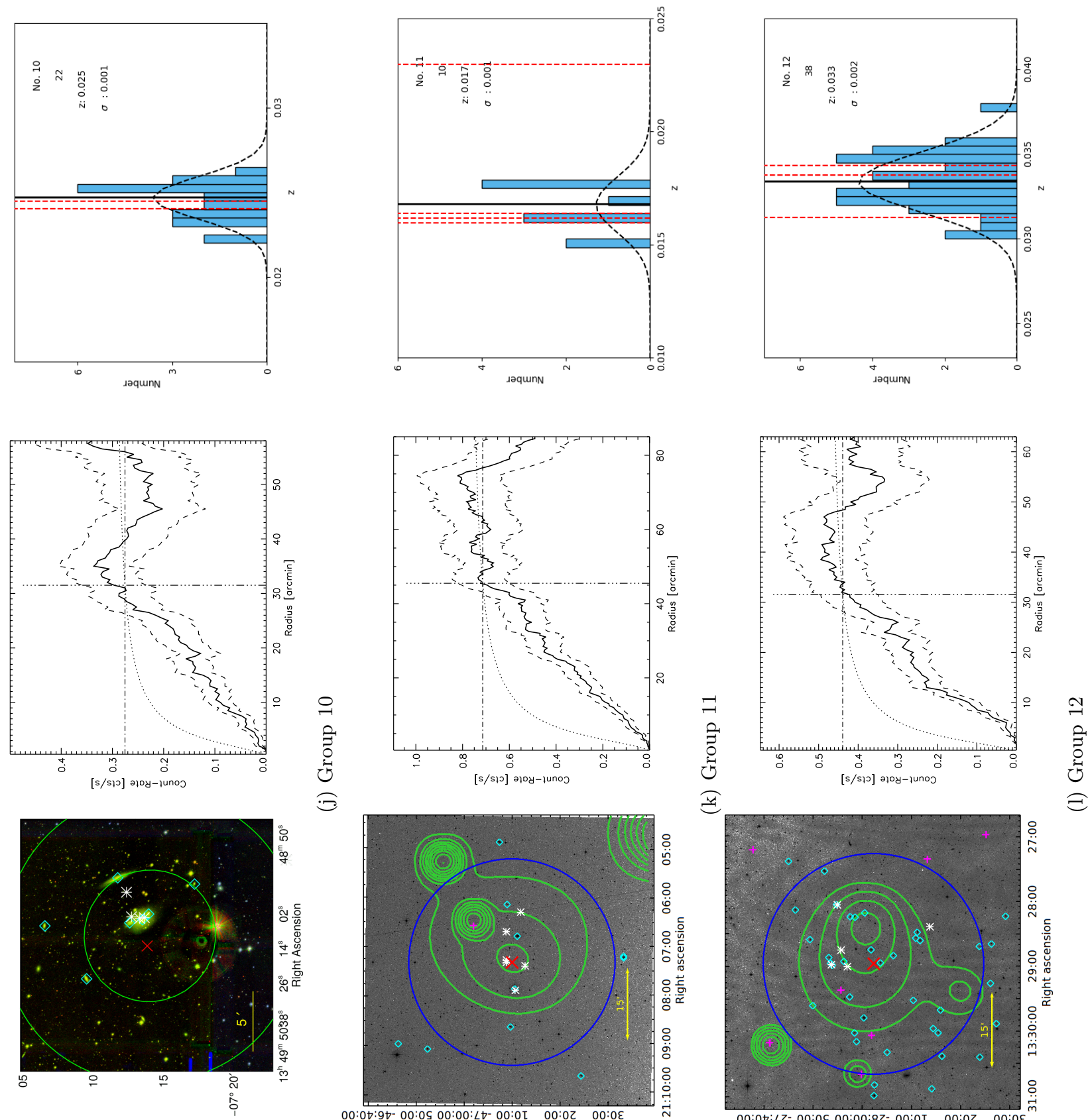

uo!̣eu!̣’əa

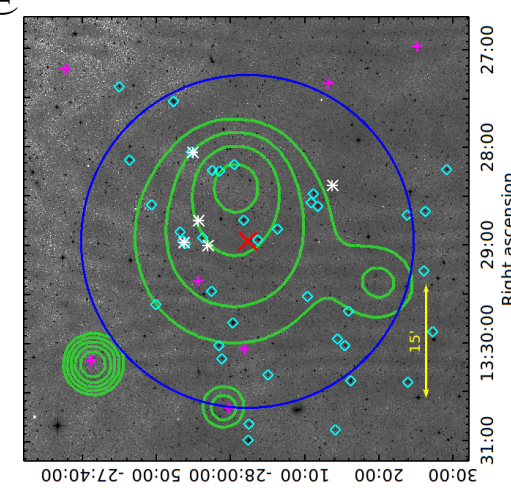

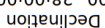
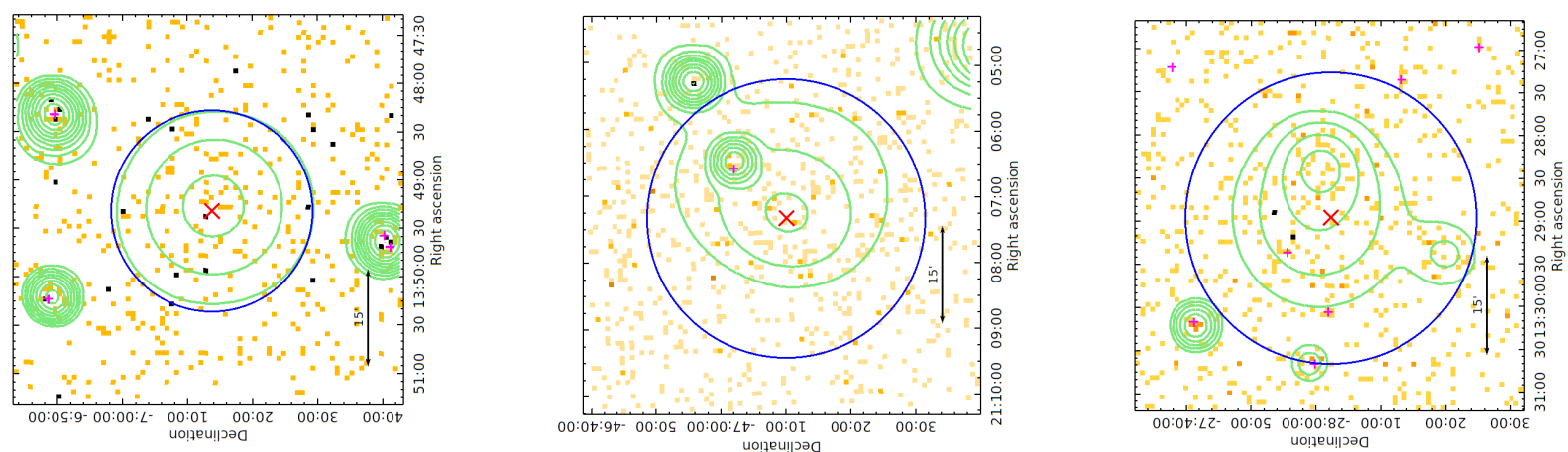

Fig. A.1. continued. 
W. Xu et al.: A sample of new extended galaxy groups from the RASS
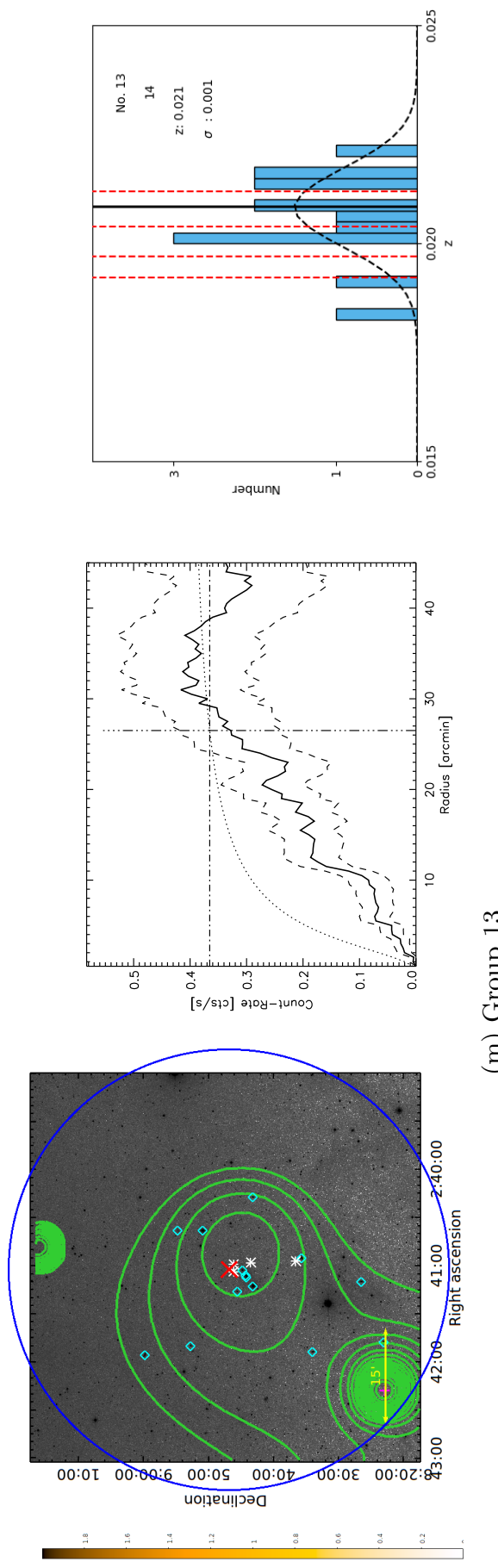

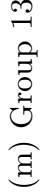

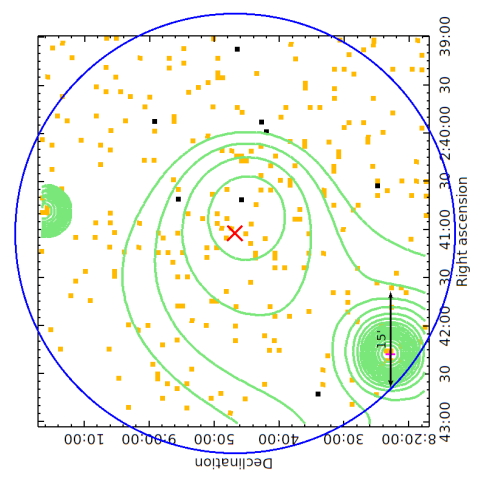

Fig. A.1. continued. 